\title{
Nouvelle approche d'analyse de la consolidation unidimensionnelle des sols argileux
}

\author{
Mohamed Ayeb ${ }^{\star}$, Mohamed Benechebli et Youssef Ayeb \\ Laboratoire d'essais, d'études et d'expertises-L3E, 4 rue El-Ordone, Hay Marrakech, Marrakech, Maroc
}

\begin{abstract}
Résumé - Dans le processus de consolidation et au regard de l'ordre de grandeur des contraintes auxquelles les sols sont soumis, les compressibilités de l'eau et des particules solides ne sont pas mises en jeu. Le volume des tassements correspond à celui de l'eau évacuée; la courbe des tassements en fonction du temps est celle de l'écoulement de l'eau : la dynamique de la consolidation est hydraulique. Ainsi selon l'état de consolidation du sol ou du protocole de chargement il peut y avoir deux ou un seul régime d'écoulement : un régime rapide et un régime lent. Les essais montrent que la fonction qui caractérise la courbe de consolidation dans son intégralité est du type hyperbolique équilatère à deux paramètres que sont la déformation finale $\varepsilon_{\infty}$ et la vitesse initiale de déformation. Les valeurs de la déformation finale obtenues par cette méthode sont conformes à celles que fournit la méthode d'Asoaka. L'introduction d'un temps adimensionnel noté $t^{*}$ permet le calcul du facteur de puissance « $m$ » utilisé pour la transposition de la courbe de consolidation à n'importe quelle longueur de chemin hydraulique. La courbe de compressibilité stabilisée (CCS) définie par les points de coordonnées $\left(\log \sigma ; \mathrm{e}_{\infty}\right)$ constitue l'enveloppe inférieure des courbes de compressibilité à différentes durées de chargement. C'est sur cette courbe que se situe les contraintes de préconsolidation caractérisant l'état de consolidation du sol. Dans ces conditions aucun accroissement de la contrainte de préconsolidation ne peut être généré par simple fluage sous une contrainte donnée et ce quelle que soit la durée de chargement considérée
\end{abstract}

Mots clés : tassement / consolidation / argiles / contrainte de préconsolidation

Abstract - New unidimensionnal consolidation analysis of clay soils. The consolidation process does not involve either the compressibility of the intergranular skeleton or that of the water. The volume of settlements corresponds to that of the evacuated water; the curve of settlements as a function of time is the counterpart of that of the flow of water: the dynamic of consolidation is exclusively hydraulic. Depending on the state of consolidation or the loading protocol, there may be two or one flow regime: a fast regime and a slow regime. The tests show that the function which characterizes the curve of consolidation in its entirety is of the equilateral hyperbolic type with two parameters which are the final deformation $\varepsilon_{\infty}$ (or the voids ratio $\mathrm{e}_{\infty}$ ) and the initial deformation velocity $* *$. The values of the final deformation obtained by this method are consistent with those provided by the method of Asoaka. The introduction of an adimensional time denoted $\mathrm{t}^{*}$ allows the calculation of the power factor " $\mathrm{m}$ " used for the transposition of the consolidation curve to any length of hydraulic path. The stabilized compressibility curve (SCC) defined by the coordinate points $(\log \sigma$; $\mathrm{e}_{\infty}$ ) constitutes the lower envelope of the compressibility curves at different loading times. It is on this curve that the preconsolidation constraints characterizing the state of consolidation of the soil lie. Consequently, no increase in the preconsolidation stress can be generated by simple creep under a given stress and this, whatever the duration of loading considered.

Keywords: settlment / consolidation / clays / preconsolidation stress

\section{Théorie de la consolidation}

L'un des problèmes les plus classiquement rencontrés en géotechnique est celui de la détermination des tassements correspondant à des diminutions d'épaisseurs des couches

\footnotetext{
$\bar{\star}$ Auteur de correspondance : md.ayeb@gmail.com
}

soumises à l'effort de compression imposé par les ouvrages projetés. La théorie de la consolidation unidimensionnelle et le principe des contraintes effectives établis par K. Terzaghi (1923) sont dès lors incontournables. Cette théorie telle qu'elle a été élaborée, porte sur la consolidation dite primaire. Elle est basée sur des considérations simplificatrices qui supposent notamment: que le sol est complètement saturé d'eau de poids volumique $\gamma_{\mathrm{w}}$, que la loi de Darcy est applicable, que la 


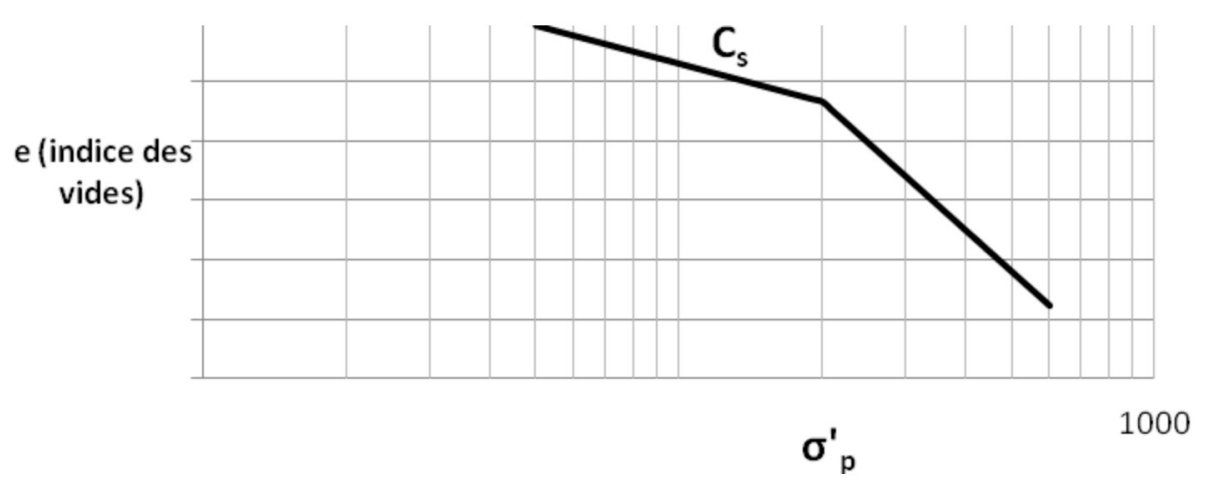

Fig. 1. Courbe de compressibilité-contrainte de préconsolidation $\sigma_{\mathrm{p}}$.

Fig. 1. Void ratio: e /log (effective stress) curve- $\sigma_{p}^{\prime}$ preconsolidation stress.

perméabilité $k$ est constante et que les déformations sont verticales et dépendent des contraintes effectives par une relation linéaire indépendante du temps. L'équation différentielle qui la caractérise est analogue à la diffusion de la chaleur (1) où $\Delta u_{(z, t)}$ est l'accroissement de la pression interstitielle à la côte $z$ et au temps $t$. $C_{v}$ est le coefficient de consolidation du sol et $E_{\text {ced }}$ module œdométrique; compte tenu des hypothèses, le coefficient de consolidation est une constante caractéristique du sol considéré.

$$
\begin{aligned}
& \frac{\partial\left(\Delta u_{(z, t)}\right)}{\partial t}=c_{v^{\prime}} \frac{\partial^{2}\left(\Delta u_{(z, t)}\right)}{\partial z^{2}} \\
& c_{v}=\frac{k \cdot E_{o e d}}{\gamma_{w}} .
\end{aligned}
$$

La solution mathématique de l'équation différentielle précédente avec les conditions aux limites et initiales conduit à définir un nombre adimensionnel appelé facteur temps désigné par $T_{v}(2)$ où $h$ est la longueur du chemin hydraulique (distance maximale entre un point quelconque du sol et le point exutoire le plus proche):

$$
T_{v}=\frac{c_{v}}{h^{2}} \cdot t=\frac{k \cdot E_{\text {oed }}}{\gamma_{w} \cdot h^{2}} \cdot t
$$

Les limites de la théorie de Terzaghi dans le cas des sols argileux compressibles ont été relevées par de nombreux chercheurs et praticiens (Buisman, 1936; Biot, 1941; Koppejan, 1948; Thompson et Palmer, 1951; Skempton et Bjerrum, 1957; Gibson et Lo, 1961; Gibson et al., 1981; Poskitt, 1969; Schlosser, 1973 ; Félix, 1980, 1981 ; Magnan, 1986; Magnan et al., 1979; Mesri, 1973; Tavenas et al., 1979; Taylor et Merchant, 1940). Des modèles faisant apparaître une deuxième phase de consolidation dénommée consolidation secondaire ont ainsi été développés. On retrouve plusieurs explications au phénomène de consolidation secondaire. Attribuée aux ruptures et aux changements d'organisation des liaisons entre les feuillets des particules pour les uns (Leonards et Ramiah, 1959 ; Barden, 1969 ; Lowe, 1974; Delage et Lefebvre, 1984; Griffiths et Joshi, 1991; Vasseur et al., 1995 ; Nakaoka et al., 2004 ; Wang et Xu, 2007), réarrangement particulaire (déformation du squelette granulaire) pour les autres (Olson, 1989). Par ailleurs, certains auteurs considèrent que les consolidations primaire et secondaire se succèdent l'une à l'autre alors que d'autres placent leur genèse en même temps. Pour Mesri et Godlewski
(1977), il n'y a pas de raison pour que les mécanismes entraînant la consolidation secondaire soient différents de ceux responsables du tassement primaire. Certains de ces modèles ont introduit des lois de comportement rhéologique de sol parfois fort complexes, mais, il faut bien admettre que, outre la difficulté de mise en œuvre de certains de ces modèles, leur «plus-value » du point de vue pratique n'a pas non plus été clairement établie. En effet la volonté de prendre en compte les lois de déformation les plus proches de la réalité a rendu parfois les modèles mathématiques inexploitables du fait de leur complexité. D'autres encore ont alimenté le développement de méthodes numériques (Abbasi et al., 2007 ; Conte et Troncone, 2007; Amiri et al., 2011).

Les essais de compressibilité à l'œdomètre constituent l'un des fondements de l'étude de la consolidation. Cet appareil reproduit, en effet, les mêmes conditions de chargement sur un échantillon de sol aux dimensions fortement réduites. Les essais permettent d'établir deux types de courbes : la courbe de compressibilité qui représente l'indice des vides (ou les déformations) en fonction du logarithme de la contrainte appliquée et la courbe de consolidation qui donne les tassements (ou les déformations) en fonction du logarithme du temps (Fig. 1 et 2).

La courbe de compressibilité permet la détermination de la contrainte historique appelée contrainte de préconsolidation $\sigma^{\prime}$ ainsi que les indices de gonflement $C_{s}$ (pente de la courbe historique) et de compression $C_{c}$ (pente de la courbe vierge). Le coefficient de consolidation $c_{v}$ et le taux de fluage $C_{\alpha}$ sont calculés à partir de la courbe de consolidation.

Le modèle de la consolidation unidimensionnelle le plus couramment utilisé s'appuie sur deux phases: une phase primaire hydraulique, caractérisée par le coefficient de consolidation $c_{v}$, qui correspond à la dissipation de l'accroissement de pression interstitielle et une phase secondaire ou de fluage, caractérisée par le taux (ou le coefficient) de fluage $C_{\alpha}$. Il existe aussi une pré-phase primaire qualifiée d'instantanée, dont l'effet est fortement amplifié par l'essai œdométrique conduit au laboratoire où la charge est appliquée instantanément (type échelon de Heaviside) sur un échantillon aux dimensions fortement réduites. Cela n'est pas le cas sur le chantier, où la mise en place du préchargement demande un certain temps qui peut aller de quelques jours à plusieurs semaines en fonction des moyens mobilisés.

Dans la pratique courante, le coefficient de consolidation $c_{v}$ est déterminé par la méthode de Casagrande (ou de Taylor). La 


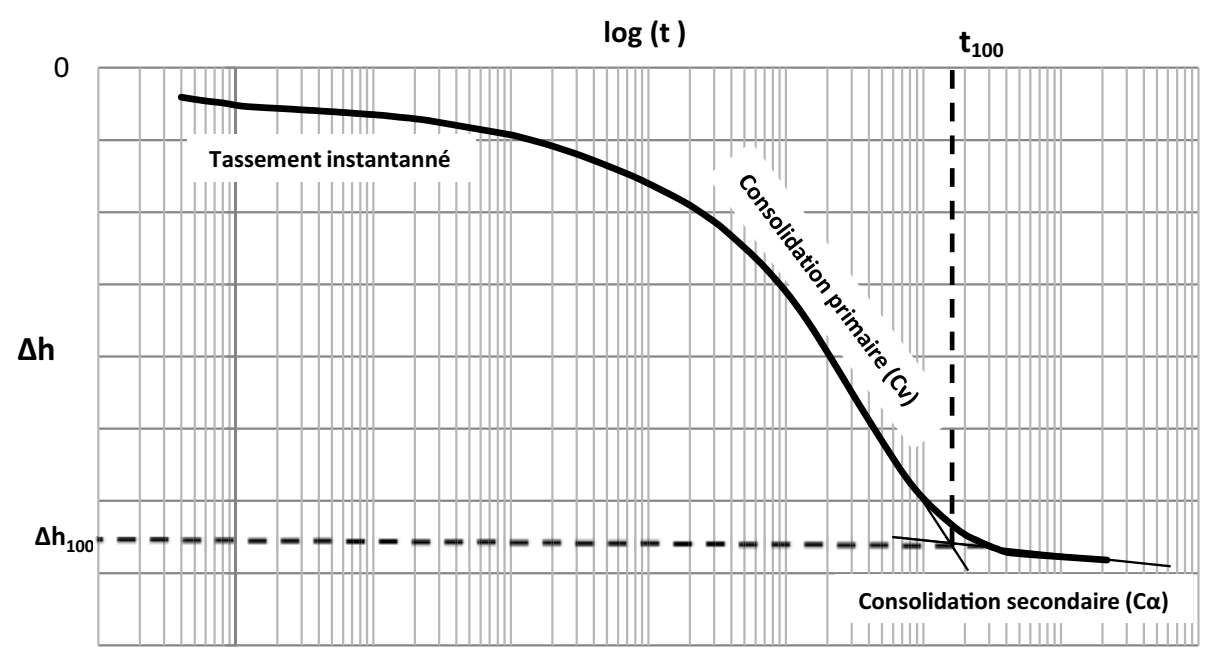

Fig. 2. Courbe de consolidation.

Fig. 2. Settlement $(\Delta h) / \log$ time curve.

courbe de consolidation en forme de « $\mathrm{S}$ penché» présente deux branches ; la première est marquée par une forte pente, la seconde branche apparaît après inflexion de la courbe avec une pente plus douce. Dans la méthode de Casagrande, l'intersection des deux droites définit le point de coordonnées $t_{100}$ et la valeur de $s_{100}$ qui correspond à la fin de la consolidation primaire où le taux de consolidation primaire atteint $100 \%$. La portion à forte pente est attribuée à la phase primaire, celle de la pente plus faible à la phase secondaire (Fig. 2). Le coefficient de consolidation $c_{v}$ est calculé en utilisant le point $t_{50}$, où le taux de consolidation primaire atteint $50 \%$ :

$$
c_{v}=\frac{0,197 h^{2}}{t_{50}},
$$

où $h$ est la longueur du chemin hydraulique.

Le taux de fluage est calculé à partir de la pente de la phase secondaire selon la méthode de Buisman et Koppejan (1948) :

$$
\frac{\Delta h}{h_{0}}=C_{\alpha} \log \left(\frac{t}{t_{100}}\right),
$$

avec, $h_{0}$ : épaisseur de la couche compressible exprimée en $\mathrm{m}$; $\Delta h$ : tassement exprimé en $\mathrm{m} ; t_{100}$ : temps marquant la fin de la consolidation primaire; $t$ : temps du développement des tassements secondaires.

\section{Caractérisation des principaux matériaux et équipements utilisés}

Les deux principaux types de matériaux utilisés, choisis pour leur différence significative d'activité argileuse, sont: la vase du canal de rocade et le ghassoul:

- la vase a été prélevée à l'extrémité aval du canal de rocade d'Al Haouz. Ce canal d'une longueur de $93 \mathrm{~km}$ achemine les eaux qui assurent une partie des besoins agricoles de la plaine du Haouz et ceux d'eau potable de la ville de Marrakech. Cette vase a une limite de liquidité de 48 et un indice de plasticité de 14 ; elle est faiblement argileuse et peu plastique. Elle présente par ailleurs un coefficient
Tableau 1. Activité argileuse.

Table 1. Clay activity.

\begin{tabular}{lll}
\hline Paramètre & Vase & Ghassoul \\
\hline Teneur en eau à la limite de liquidité $w_{L}$ en $\%$ & 48 & 137 \\
Teneur en eau à la limite de plasticité $w_{P}$ en $\%$ & 34 & 54 \\
Indice de plasticité $I_{P}=w_{L}-w P$ & 14 & 83 \\
$\mathrm{PA}=$ pourcentage de la fraction argileuse & 44 & 30 \\
$(\mathrm{dim}<2 \mu \mathrm{m})$ & & \\
Coefficient d'activité argileuse $=\mathrm{I}_{\mathrm{P}} / \mathrm{PA}$ & 0,32 & 2,77 \\
Classe de Skempton & Inactif & Très actif
\end{tabular}

d'activité argileuse de 0,32 , ce qui la situe dans la classe des sols inactifs selon Skempton (Tab. 1);

- le ghassoul est une argile connue pour ses différentes utilisations esthétiques: traitement des cheveux et de la peau. Le ghassoul a une limite de liquidité de 137 et un indice de plasticité de 83 ; c'est un matériau très argileux et très plastique. Il présente un coefficient d'activité argileuse de 2,3 , ce qui le situe dans la classe des sols très actifs selon Skempton (Tab. 1).

Les courbes granulométriques de ces deux matériaux sont représentées sur la figure 3 .

Afin de disposer d'échantillons homogènes, les matériaux ont été prélevés dans le terrain puis reconditionnés pour atteindre la teneur en eau à la limite de liquidité $w_{L}$ (Tab. 1).

Deux œdomètres ont été utilisés pour la réalisation des essais. Le premier, de type classique, est drainé des deux faces, avec une longueur de chemin hydraulique de $1 \mathrm{~cm}$; le second n'est drainé que par sa face supérieure, la face inférieure étant munie d'un capteur de pression; il présente une longueur de chemin hydraulique de $2 \mathrm{~cm}$. Les mesures de pression peuvent être réalisées avec des pas de temps ajustables et pouvant aller jusqu'à $2 / 10^{\mathrm{e}}$ de seconde. Les tests effectués lors d'essais non drainés sur les échantillons, avec des chargements de 200,400 puis $600 \mathrm{kPa}$, ont montré la concordance des réponses fournies par le capteur de pression avec les contraintes appliquées. 


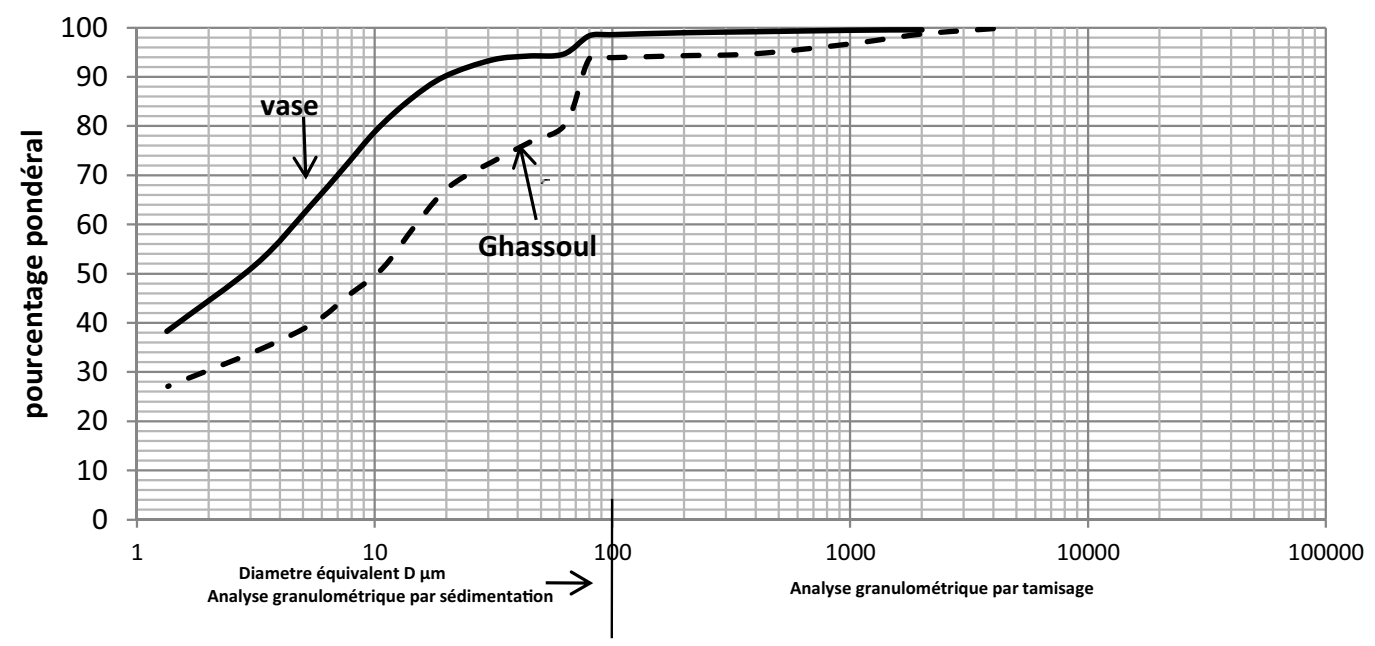

Fig. 3. Courbes granulométriques de la vase et du ghassoul.

Fig. 3. Grading curves for mud and ghassoul.
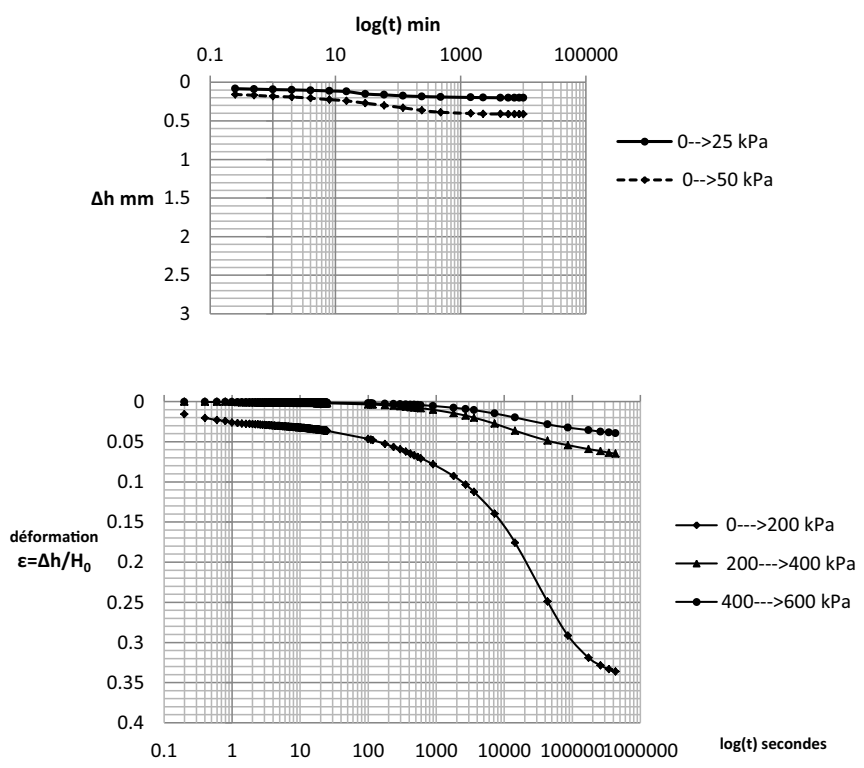

Fig. 4. a : Ghassoul surconsolidé $\left(\sigma_{\mathrm{p}}^{\prime}=150 \mathrm{kPa}\right.$, chargement pendant 7 jours); $b$ : Ghassoul normalement consolidé avec trois chargements successifs de 5 jours de durée et $H_{\mathrm{o}}$ correspond à la nouvelle hauteur de l'éprouvette au début du nouveau chargement.

Fig. 4. $a$ : Overconsolidated ghassoul $-\sigma^{\prime}{ }_{p}=150 \mathrm{kPa}$-loading time $=$ 7 days; $\Delta$ hesttlement; $b$ : Normally consolidated ghassoul-three successive loads-loading time $=5$ days each. $H_{o}$ height at the beginning of the new loading.

\section{Limites de l'approche usuelle actuelle}

On relèvera que le $t_{100}$ utilisé par les méthodes de Casagrande et de Buisman et Koppejan correspond au temps où le taux de consolidation de la phase primaire a atteint $100 \%$, or, selon la théorie, ce taux n'est atteint qu'au bout d'un temps infini.

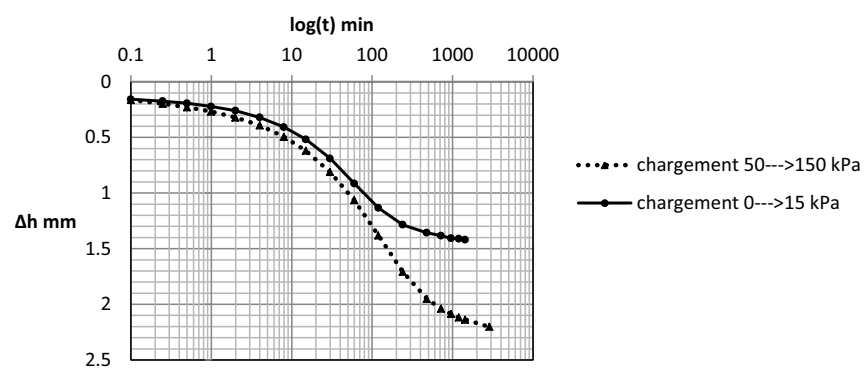

Fig. 5. Ghassoul remanié avec 2 paliers de chargement de 2 jours de durée.

Fig. 5. Two loadings increments of ghassoul; duration 2 days each.

L'application de ces méthodes est conditionnée par le fait que la courbe de consolidation présente une forme en « $\mathrm{S}$ » avec notamment une phase primaire bien marquée et qui est suivie par un point d'inflexion. Or, quand ces conditions font défaut, la mise en œuvre de ces méthodes s'avère délicate voire impossible. C'est le cas par exemple de certains essais conduits sur des sols surconsolidés et pour lesquels la contrainte appliquée est inférieure à la contrainte de préconsolidation (Fig. $4 \mathrm{a}, \sigma_{\mathrm{p}}=150 \mathrm{kPa}$ ) ou dans le cas d'une série de chargements avec un palier constant sur un sol normalement consolidé (Fig. 4b) ainsi que des courbes établies par Sridharan et Sreepada Rao (1981). Sur les figures, les courbes de tassements commencent à 0 à chaque nouveau chargement.

Le coefficient de consolidation $c_{v}$ n'est pas un invariant du sol considéré. Il dépend de la charge appliquée. Ainsi, les chargements du ghassoul de 0 à $15 \mathrm{kPa}$ et de 50 à $150 \mathrm{kPa}$ conduisent à un $c_{v 15}$ de $9,5.10^{-9} \mathrm{~m}^{2} / \mathrm{s}$ dans le premier cas; et à un $c_{v 150}$ de $2,9.10^{-9} \mathrm{~m}^{2} / \mathrm{s}$ dans le second (Fig. 5).

Aussi, les essais conduits sur deux échantillons du même matériau soumis aux mêmes conditions de chargement mais avec des longueurs de chemin hydraulique différentes ne présentent pas le même coefficient de consolidation $c_{v}$. Dans le cas de la vase avec un chargement de 0 à $100 \mathrm{kPa}$ durant cinq jours, en drainage unilatéral, le $c_{v}$ est de $7,5.10^{-8} \mathrm{~m}^{2} / \mathrm{s}$; il est de 


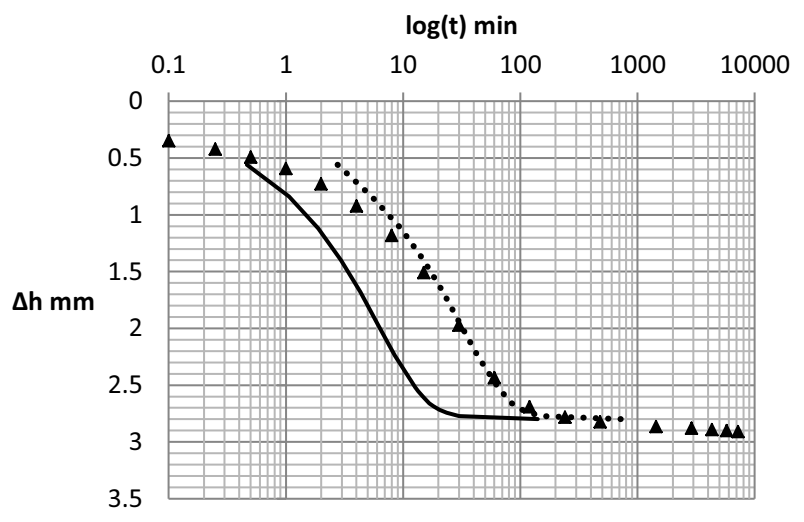

- drainage unilatéral/halfclosed layer

ajustement avec Cv drainage unilatéral/Adjustement with Cv half-closed layer

- ajustement avec Cv drainage bilatéral/Adjustement with $\mathrm{Cv}$ open layer

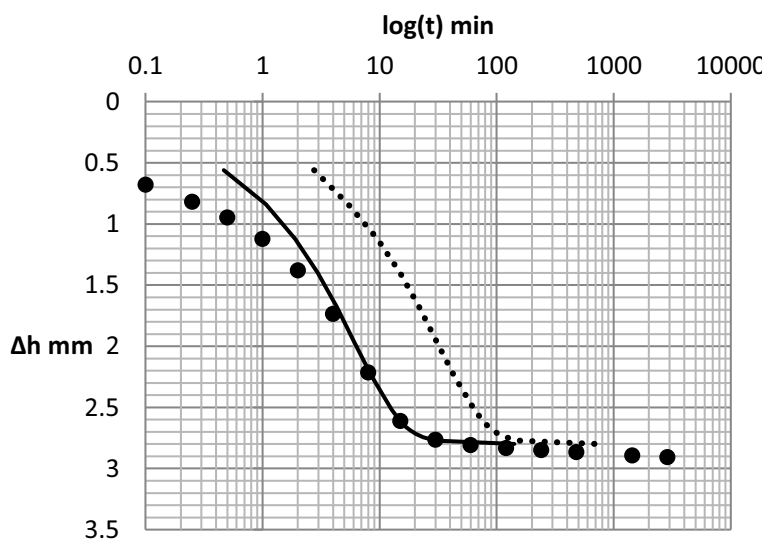

- drainage bilatéral/open layer

— ajustement avec Cv drainage bilatéral/Adjustement with Cv open layer

..... ajustement avec Cv drainage unilatéral/Adjustement with $\mathrm{Cv}$ half-closed layer

Fig. 6. a : Vase en drainage unilatéral : chargement $0 \rightarrow 100 \mathrm{kPa}$, durée 5 jours ; b : Vase en drainage bilatéral : chargement $0 \rightarrow 100 \mathrm{kPa}$, durée 5 jours.

Fig. 6. a: Mud: half-closed layer; load $0 \rightarrow 100 \mathrm{kPa}$-duration 5 days; $b:$ Mud: open layer; load $0 \rightarrow 100 \mathrm{kPa}-$ duration 5 days.

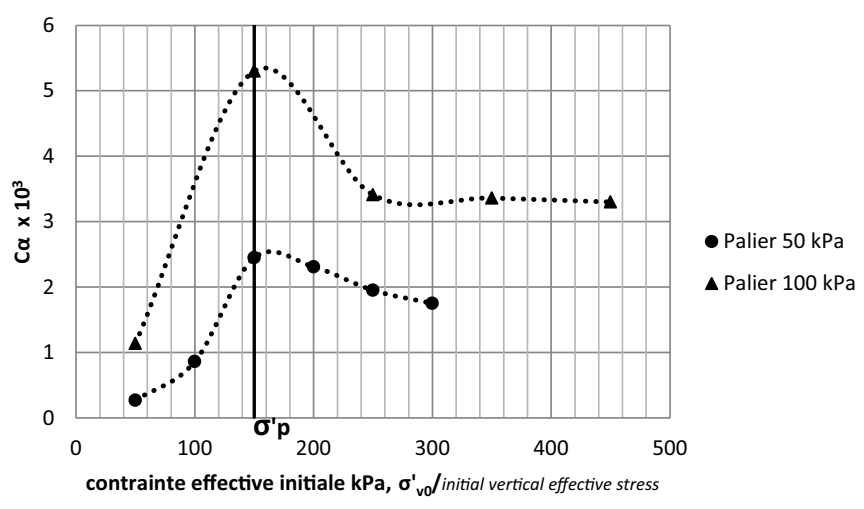

Fig. 7. Variation du coefficient de fluage $C_{\alpha}$ en fonction de $\sigma^{\prime}{ }_{\text {vo }}$ et du palier de chargement $\Delta \sigma$.

Fig. . Variation of the secondary compression index $C_{\alpha}$ as function of the initial vertical effective stress and of the loading increment $\Delta \sigma$.

$1,1 \cdot 10^{-7} \mathrm{~m}^{2} / \mathrm{s}$ en drainage bilatéral. L'adéquation de l'ajustement aux points expérimentaux dépend $\mathrm{du} c_{v}$ considéré (Fig. 6a et b).

À l'instar du $c_{v}$, le coefficient $C_{\alpha}$ caractéristique de la phase secondaire (Philipponnat et Hubert, 2011) n'est pas non plus un invariant du sol considéré. Il dépend non seulement du palier de chargement mais aussi de la contrainte effective initiale $\sigma^{\prime}{ }_{\mathrm{v} 0}$. Il atteint son maximum autour de la contrainte de préconsolidation. La figure 7 reproduit les variations du coefficient $C_{\alpha}$ en fonction de la contrainte effective initiale $\sigma^{\prime}{ }_{\mathrm{v} 0}$ pour deux séries de chargement de la vase préconsolidée à $150 \mathrm{kPa}$ avec un palier de $50 \mathrm{kPa}$ pour la première série et de $100 \mathrm{kPa}$ pour la seconde série. Les chargements de la première série sont: 50, 100, 150, 200, 250,300 et enfin $350 \mathrm{kPa}$; ceux de la seconde série sont 50, 150, 250,350 et $450 \mathrm{kPa}$. La durée de chaque chargement est de $24 \mathrm{~h}$.

Caractérisant la phase secondaire, le taux de fluage est impacté pourtant par les conditions de drainage. En effet, sa valeur croît avec la longueur du chemin hydraulique. Cela est confirmé par les essais réalisés sur la vase en drainage unilatéral et bilatéral (Fig. 8). Pour les deux longueurs de chemin hydraulique, le chargement de $100 \mathrm{kPa}$ a été appliqué durant 5 jours. Le $C_{\alpha}$ drainage unilatéral est de $4,0.10^{-3}$ alors que le $C_{\alpha}$ drainage bilatéral est de $2,6.10^{-3}$.

Les essais réalisés à l'aide d'un œdomètre équipé d'un capteur de pression montrent qu'au point considéré comme marquant la fin de la phase primaire, la pression interstitielle n'est pas nulle et que, en conséquence, une partie de la courbe imputée à la phase secondaire relève de la phase primaire (Fig. 9a et b). La pression résiduelle à $t_{100}$ est de $32 \mathrm{kPa}$ dans le cas de la vase et de $60 \mathrm{kPa}$ dans le cas du ghassoul. 


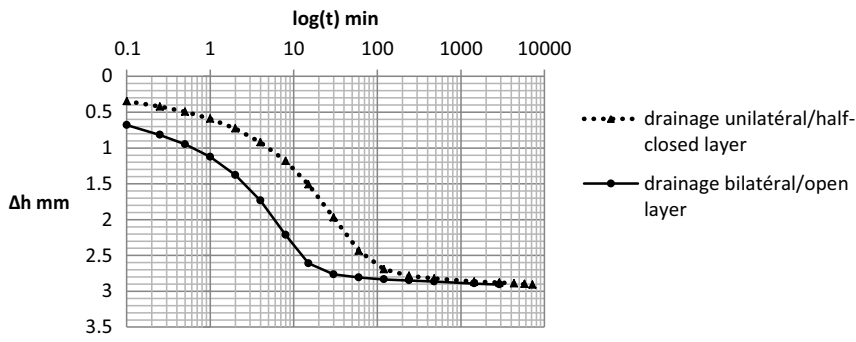

Fig. 8. Dépendance du coefficient de fluage $C_{\alpha}$ des conditions de drainage-Vase: chargement de $100 \mathrm{kPa}$ en drainage unilatéral et bilatéral, durée 5 jours.

Fig. 8. Dependence of the secondary compression index $C_{\alpha}$ of drainage conditions-Mud-load 5 days $0 \rightarrow 100 \mathrm{kPa}$; open and half-closed layer.
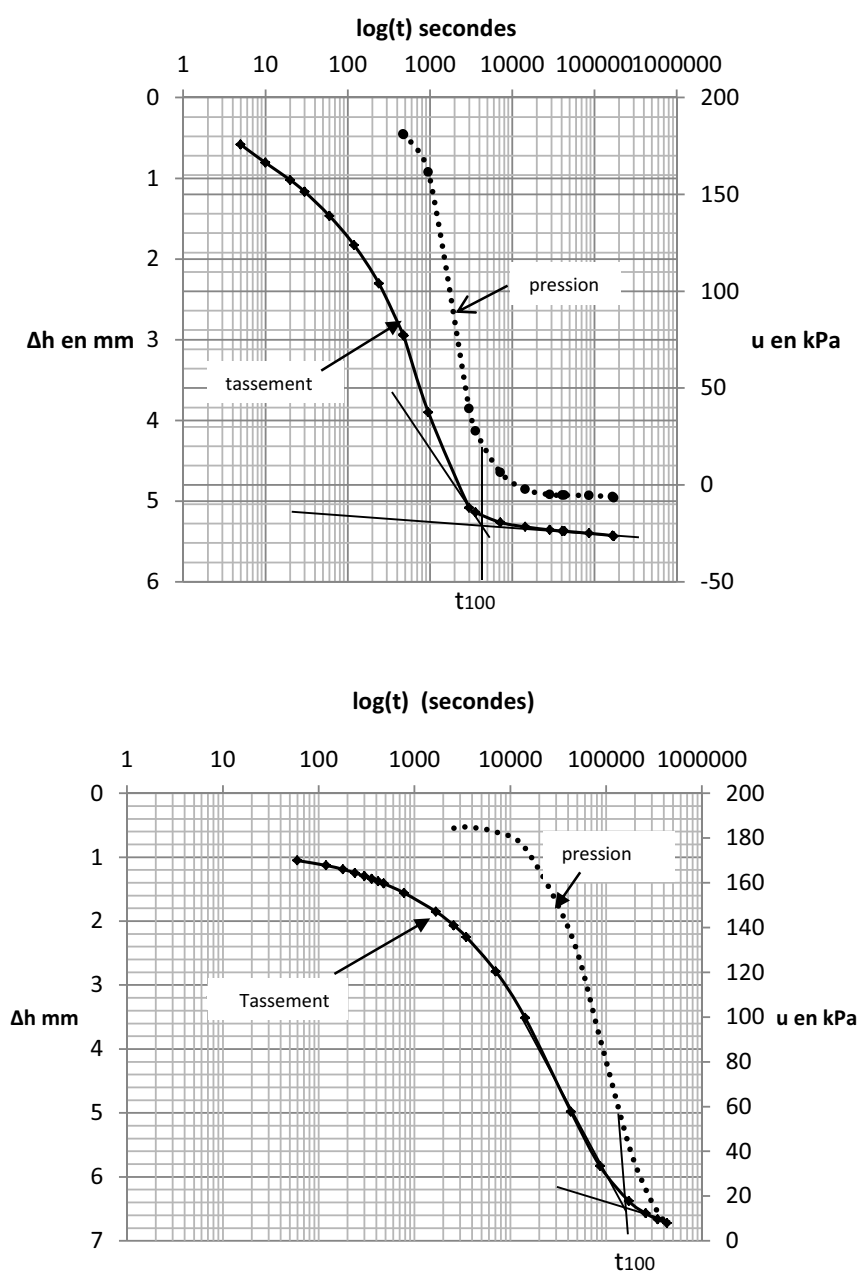

Fig. 9. a: Consolidation avec suivi de la pression interstitielle de la vase : chargement $0 \rightarrow 200 \mathrm{kPa}$ pendant 2 jours. Au point $t_{100}$ la pression résiduelle est de $32 \mathrm{kPa}$; $\mathrm{b}$ : Consolidation avec suivi de la pression interstitielle du ghassoul : chargement $0 \rightarrow 200 \mathrm{kPa}$ pendant 2 jours. Au point $t_{100}$ la pression résiduelle est de $60 \mathrm{kPa}$.

Fig. 9. a: Consolidation with pore-water pressure control in mud; load $0 \rightarrow 200 \mathrm{kPa} 2$ days; at $t_{100}$ the residual pore-water pressure is $32 \mathrm{kPa}$; $b$ : Consolidation with pore-water pressure control in ghassoul; load $0 \rightarrow 200 \mathrm{kPa} 2$ days; at $t_{100}$ the residual pore-water pressure is $60 \mathrm{kPa}$.

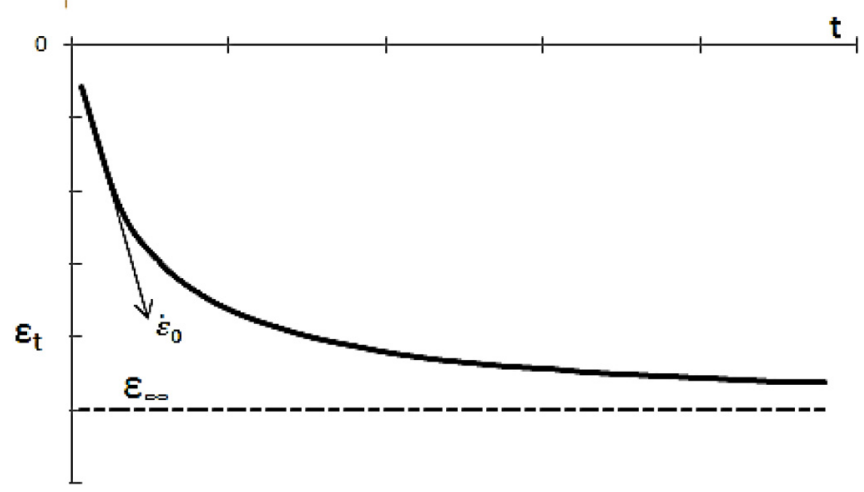

Fig. 10. Courbe de consolidation en échelle arithmétique.

Fig. 10. Consolidation graph in arithmetic time scale.

\section{Nouvelle approche d'analyse de la consolidation unidimensionnelle}

Les limites et les difficultés que présente l'approche segmentée usuelle amènent à la recherche d'autres méthodes permettant de mieux appréhender le phénomène de la consolidation. Dans le cadre de ces travaux, nous avons adopté une méthode expérimentale qui traite la courbe de consolidation dans sa globalité et qui s'appuie sur sa dimension hydraulique. Cette approche qui vise à dégager une méthode simple et pratique pour le calcul des tassements à différents horizons de temps s'appuie sur les considérations suivantes:

- au regard de l'ordre de grandeur des contraintes auxquelles les sols sont soumis, les compressibilités de l'eau et des particules solides ne sont pas mises en jeu. Le volume des tassements correspond à celui de l'eau évacuée; la courbe des tassements en fonction du temps reflète celle de l'écoulement de l'eau;

- même en phase de fluage, le processus de consolidation s'interrompt à l'arrêt du drainage; cela confirme que la dynamique de la consolidation est hydraulique;

- la représentation usuelle des tassements ou des déformations $\varepsilon$ en fonction du temps faite en adoptant une échelle logarithmique en abscisse (Fig. 2), présente l'avantage de pouvoir représenter des temps allant de la minute jusqu'à plusieurs jours (voire même plusieurs mois). Cette distorsion de temps présente en revanche l'inconvénient d'amplifier le visuel des pentes des premières décades siège de la phase de consolidation primaire. Or, dans la représentation en coordonnées linéaires (temps, déformation) (Fig. 10); la courbe de consolidation présente une allure hyperbolique et aucune des phases attribuées à la consolidation primaire et secondaire ne peut être mise en évidence. Le point de coordonnées $\left(t_{100}, \varepsilon_{100}\right)$ marque la séparation de deux régimes d'écoulement: un régime d'écoulement rapide où les vitesses de tassement sont notables (c'est la phase primaire) et un régime d'écoulement lent et où $a$ contrario les vitesses de tassement sont relativement lentes (c'est la phase secondaire ou le fluage). Le temps nécessaire pour atteindre un tassement donné $\Delta h$, est beaucoup plus rapide en phase primaire qu'en phase secondaire (Fig. 22). 


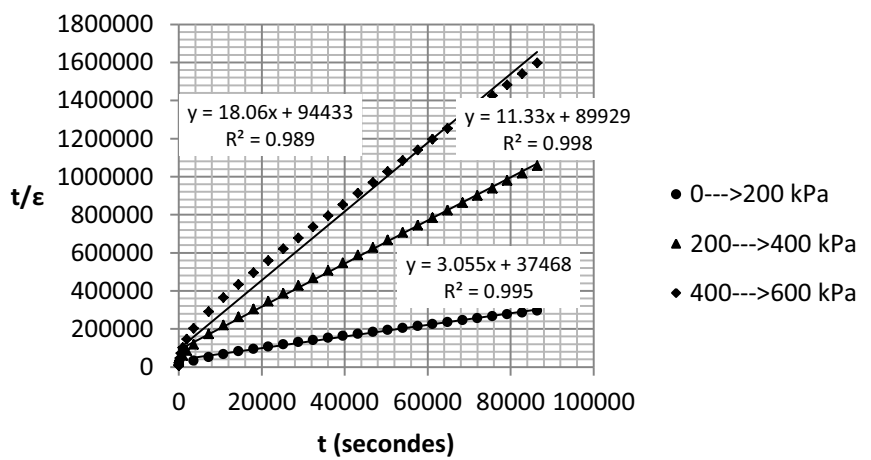

Fig. 11. Détermination des paramètres d'ajustement du ghassoul pour les trois chargements successifs de 5 jours de durée chacun.

Fig. 11. Determining ghassoul adjustment parameters for the three successive loadings of five days duration each.

Les essais à l'œdomètre effectués sur deux types de sol présentant des niveaux d'activité argileuse différents ont permis de confirmer que la fonction hyperbolique équilatère s'ajuste à la courbe de consolidation (Sridharan et Sreepada Rao, 1981, 1987; Tan et al., 1991; Al Shamrani, 2004). La loi de consolidation qui décrit parfaitement l'intégralité du phénomène est caractérisée par deux grandeurs : la déformation finale $\varepsilon_{\infty}$ et la vitesse initiale de déformation $\dot{\varepsilon}_{0}$. La loi de consolidation se présente sous la forme (5):

$$
\varepsilon_{t}=\frac{t}{\frac{1}{\dot{\varepsilon}_{0}}+\frac{t}{\varepsilon_{\infty}}} .
$$

La représentation $\frac{t}{\varepsilon_{t}}=f(t)$, permet d'ajuster une droite dont la pente est l'inverse de $\varepsilon_{\infty}$ et l'ordonnée à l'origine est l'inverse de $\dot{\varepsilon}_{0}$.

Compte tenu de la nature même de la consolidation, l'écoulement engendré par l'accroissement de contraintes totales est du type paradoxal: car il génère les propres conditions de sa résorption. En effet, les tassements résultant de l'évacuation de l'eau, réduisent les sections de passage et accroissent en conséquence les pertes de charge ce qui ralentit les vitesses d'écoulement et donc celles des tassements.

\section{Essais et résultats}

Chacun des deux matériaux est reconditionné au départ de chaque série de mesures à la limite de liquidité $w_{L}$. Les chargements successifs, d'une durée de 5 jours chacun pour le ghassoul et de 2 jours pour la vase, sont de $200 \mathrm{kPa}$, suivi de $400 \mathrm{kPa}$ et enfin de $600 \mathrm{kPa}$. Les essais en état surconsolidé (Fig. 16a-d) ont été effectués avec les mêmes chargements pour une durée de 2 jours ; la contrainte de préconsolidation est de $600 \mathrm{kPa}$.

On procède à la mesure du tassement et du temps correspondant. Dans l'œdomètre muni de capteur de pression, en plus du temps et du tassement, la mesure de la pression est aussi effectuée avec un pas de temps ajustable (minimum $2 / 10^{\mathrm{e}}$ de seconde).

Pour la mise en œuvre de la méthode hyperbolique, seules les mesures effectuées durant les 24 premières heures ont été utilisées. Les déformations $\varepsilon_{\infty}$ calculées ont ensuite été comparées à celles mesurées en fin d'essai (Tab. 3, 4 et 5$)$

Les figures 11 et 12 représentent $t / \varepsilon=f(t)$ pour le ghassoul et la vase. Elles permettent de déterminer par ajustement

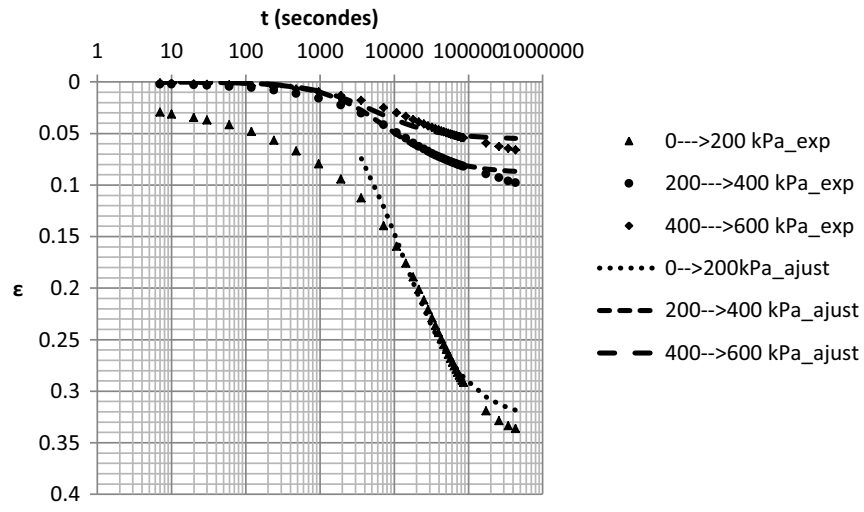

Fig. 12. Détermination des paramètres d'ajustement de la vase pour les trois chargements.

Fig. 12. Determining mud adjustment parameters for the three successive loadings of two days duration each.

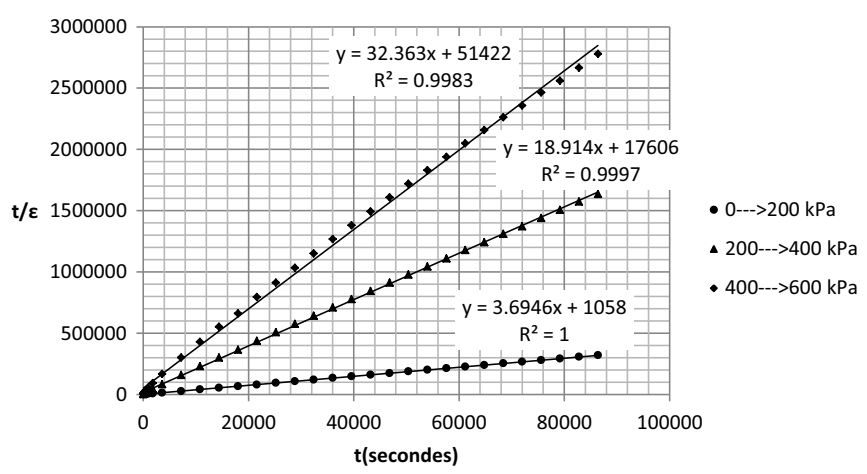

Fig. 13. Ajustement de la loi hyperbolique pour les trois chargements successifs du ghassoul.

Fig. 13. Hyperbolic fit for the three successive loadings of ghassoul.

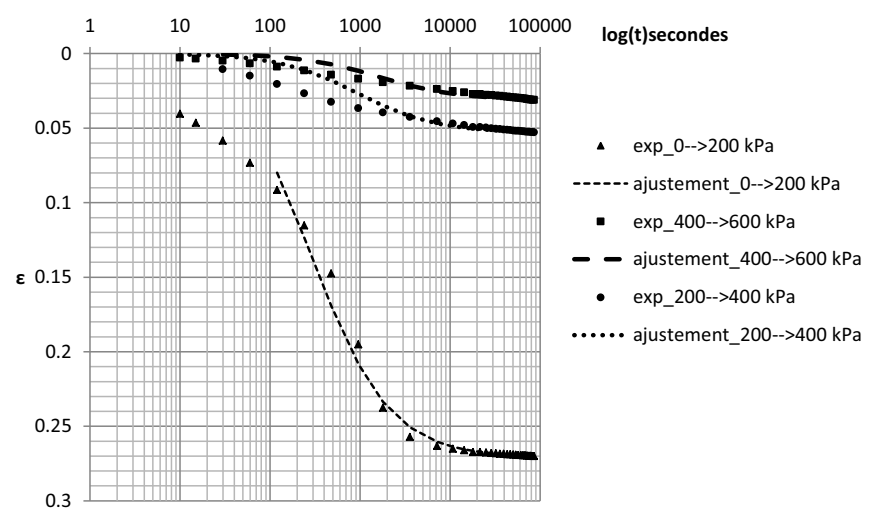

Fig. 14. Ajustement de la loi hyperbolique pour les trois chargements de la vase.

Fig. 14. Hyperbolic fit for the three successive loadings of mud.

linéaire les deux paramètres $\varepsilon_{\infty}$ et $\dot{\varepsilon}_{0}$ pour les trois chargements. Les excellentes valeurs des coefficients de détermination permettent d'apprécier la bonne qualité des ajustements. Les figures 13 et 14 reproduisent les courbes classiques de consolidation $\varepsilon=\varepsilon(\log t)$ et les ajustements respectifs pour le ghassoul et la vase. 
Tableau 2. Vitesses de déformations initiales et déformations finales.

Table 2. Initial strain rate and final deformation.

\begin{tabular}{|c|c|c|c|c|c|c|}
\hline$\frac{\text { Chargement }}{\text { Matériau }}$ & \multicolumn{2}{|c|}{$0 \rightarrow 200 \mathrm{kPa}$} & \multicolumn{2}{|c|}{$200 \longrightarrow 400 \mathrm{kPa}$} & \multicolumn{2}{|c|}{$400 \longrightarrow 600 \mathrm{kPa}$} \\
\hline & $9,45 \cdot 10^{-4}$ & $2,67 \cdot 10^{-5}$ & $5,68 \cdot 10^{-5}$ & $1,11.10^{-5}$ & $1,94 \cdot 10^{-5}$ & $1,06 \cdot 10^{-5}$ \\
\hline$\varepsilon_{\infty \text { hyperbolique }}$ & 0,27 & 0,33 & 0,05 & 0,09 & 0,03 & 0,06 \\
\hline
\end{tabular}

Tableau 3. Vitesses de déformations initiales et déformations finales en état surconsolidé.

Table 3. Initial strain rate and final deformation in overconsolidated state.

\begin{tabular}{lllll}
\hline Chargement & \multicolumn{2}{c}{$0 \rightarrow 200 \mathrm{kPa}$} & \multicolumn{2}{c}{$200 \rightarrow 400 \mathrm{kPa}$} \\
\hline Matériau & Vase & Ghassoul & Vase & Ghassoul \\
$\dot{\varepsilon}_{0} \cdot\left(\mathrm{s}^{-1}\right)$ & $1,57.10^{-4}$ & $1,37.10^{-5}$ & $2,61.10^{-5}$ & $1,02.10^{-5}$ \\
$\varepsilon_{\infty}$ hyperbolique & 0,028 & 0,050 & 0,011 & 0,030 \\
$\varepsilon_{24 \mathrm{~h}}$ & 0,028 & 0,048 & 0,011 & 0,030 \\
$\varepsilon_{2 \mathrm{j}}$ & 0,029 & 0,049 & 0,011 & 0,032 \\
\hline
\end{tabular}

\section{Paramètres d'ajustement de la loi hyperbolique $\dot{\varepsilon}_{0}$. et $\varepsilon_{\infty}$}

La vitesse de déformation initiale $\dot{\varepsilon}_{0}$. (Tab. 2 et 3) apparaît comme une des grandeurs caractéristiques du matériau qui, à l'instar de la perméabilité, régit les conditions d'écoulement provoquées par l'accroissement de charge. Elle décroît avec la plasticité du matériau. Ainsi, $\dot{\varepsilon}_{0}$. de la vase est supérieure à celle du ghassoul. Pour un même matériau, les vitesses de déformation initiales décroissent lors de l'application d'une série de chargement croissante. La vitesse de déformation dépend aussi de l'état de consolidation du matériau, sa valeur en état surconsolidé ${ }^{1}$ est inférieure à celle de l'état normalement consolidé (Fig. 15a-d; Tab. 3).

Note : $\varepsilon_{\infty}$ (Tab. 2, 3, 4 et 5) représente la déformation finale qui détermine un état d'équilibre avec la contrainte appliquée $\sigma_{\mathrm{t}}$. Elle ne correspond pas toujours à $\varepsilon_{24 \mathrm{~h}}$ comme le montrent les résultats des essais sur le ghassoul du tableau 4. Pour les sols peu plastiques, l'écart entre les deux valeurs est insignifiant. En revanche pour le ghassoul, matériau très plastique et qui présente une argilosité élevée, l'écart entre les deux déformations ne peut être négligé. Pour un même chargement, la déformation finale $\varepsilon_{\infty}$ est plus importante pour le matériau présentant le coefficient d'activité argileuse le plus élevé (Tab. 2).

$\varepsilon_{\infty}$ dépend de la nature du sol, de son patrimoine historique (nature et contrainte de préconsolidation $\sigma_{\mathrm{p}}^{\prime}$ notamment), actuel $\left(\sigma^{\prime}{ }_{v 0}\right.$, contrainte initiale) et futur, $\Delta \sigma$ (accroissement de contrainte). En plus des paramètres précédents, dépend de la longueur du chemin hydraulique (Fig. 16 et Tab. 2, 3, 6a et 6b).

\footnotetext{
${ }^{1}$ En état surconsolidé (contrainte de préconsolidation de $600 \mathrm{kPa}$ ), les deux premières séries de chargement ont été effectuées durant 48 heures.
}
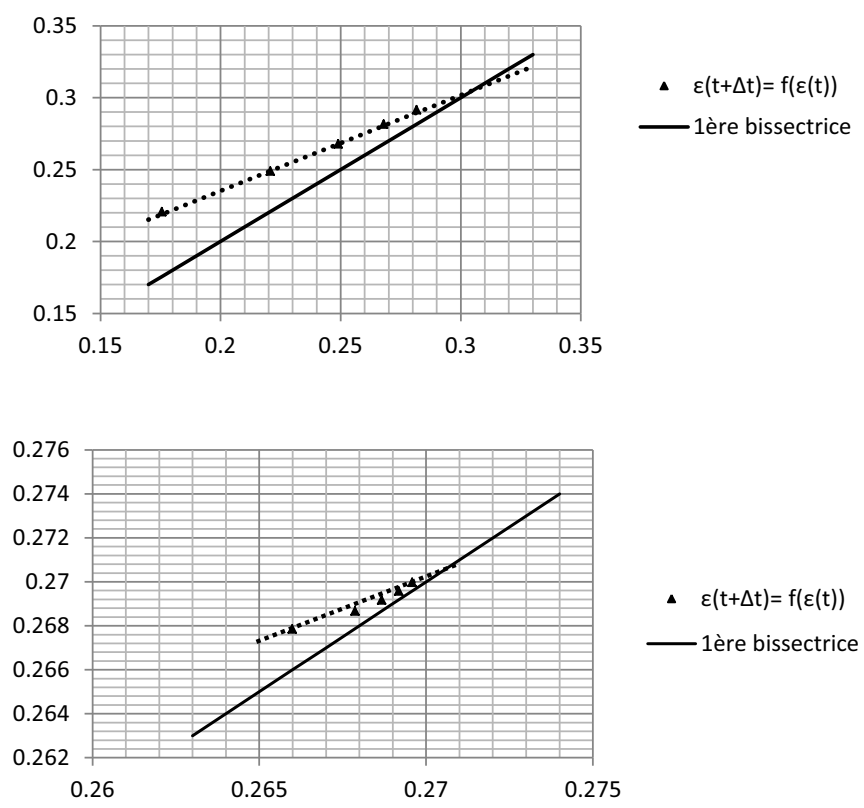

Fig. 15. a : Construction graphique d'Asoaka : ghassoul : chargement $0 \rightarrow 200 \mathrm{kPa} ; \mathrm{b}$ : Construction graphique d'Asoaka : Vase : chargement $0 \rightarrow 200 \mathrm{kPa}$.

Fig. 15. a: Graphic construction of Asoaka-ghassoul: loading $200 \mathrm{kPa}$; b: Graphic construction of Asoaka-mud: loading $200 \mathrm{kPa}$.

\section{Comparaison des déformations finales avec la méthode graphique d'Asoaka}

Afin de pouvoir vérifier la pertinence des valeurs de $\varepsilon_{\infty}$ obtenues par la méthode hyperbolique, il a été procédé à leur comparaison avec celles obtenues par la méthode graphique d'Asoaka (Asaoka, 1978; Magnan et Deroy, 1980 ; Baguelin, 1999; Tan et Chew, 1996).

La construction graphique d'Asoaka consiste à reporter les déformations successives espacées par un intervalle de temps constant $\Delta t: \varepsilon(t+\Delta t)=f(\varepsilon(t))$; la droite qui s'ajuste au mieux (au sens des moindres carrés) aux points $P_{\mathrm{i}}$ ainsi obtenus intercepte la première bissectrice à la valeur de la déformation finale $\varepsilon_{\infty}$ (Fig. 17a et b). Il faut toutefois signaler les difficultés dans la mise en œuvre de cette méthode et qui sont de deux sortes :

- les mesures sont rarement effectuées avec un intervalle de temps constant ce qui nécessite de procéder à des interpolation ; 

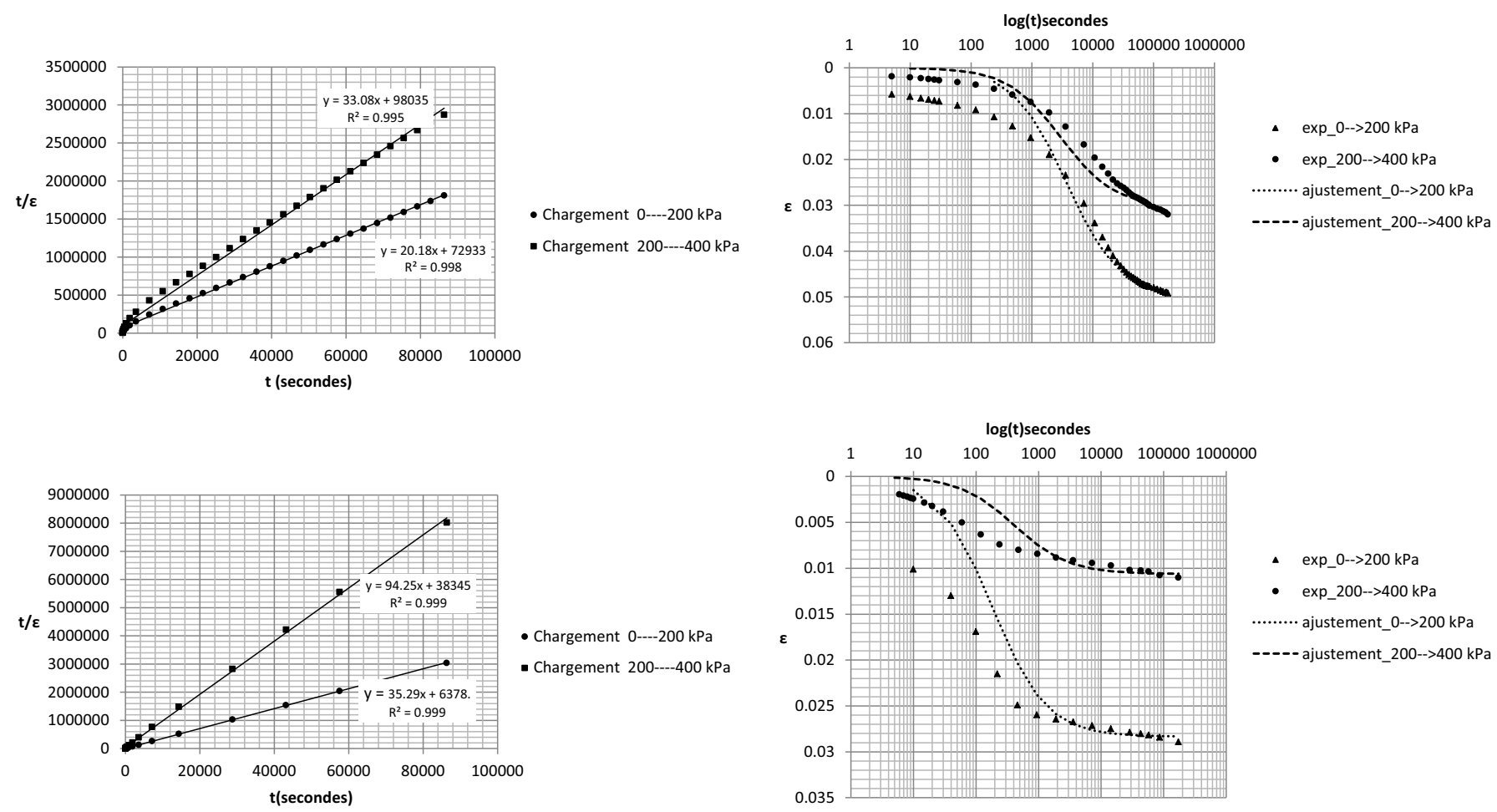

Fig. 16. a: Détermination des paramètres d'ajustement du ghassoul en état surconsolidé $(\sigma$ ’ $p=600 \mathrm{kPa})$ durée de chargement 2 jours; $\mathrm{b}$ : Ajustement de la loi hyperbolique pour les deux chargements du ghassoul ; $\mathrm{c}$ : Détermination des paramètres d'ajustement de la vase en état surconsolidé $\left(\sigma^{\prime} \mathrm{p}=600 \mathrm{kPa}\right)$. Durée de chargement 2 jours; $\mathrm{d}$ : Ajustement de la loi hyperbolique pour les deux chargements de la vase.

Fig. 16. a: Determining adjustment parameters of overconsolidated ghassoul for two successive loadings of 2 days duration each $-\sigma^{\prime} p=600 \mathrm{kPa} ; b$ : Hyperbolic fit for the two successive loadings of overconsolidated ghassoul; c: Determining adjustment parameters of overconsolidated mud for two successive loadings of 2 days duration each $-\sigma$ ' $p=600 \mathrm{kPa}$; d: Hyperbolic fit for the two successive loadings of overconsolidated mud.

- les fluctuations inhérentes aux dispositifs de mesures qui peuvent introduire des biais dans la tendance générale (Fig. $18 b$ et d).

Les calculs des déformations finales ont été effectués par la méthode hyperbolique et par celle d'Asoaka. Les tableaux 4 et 5 comportent en plus de ces valeurs calculées respectivement pour le ghassoul et la vase, les déformations mesurées au bout de $24 \mathrm{~h}$ et de 5 jours pour le ghassoul et de 2 jours pour la vase.

Les résultats fournis par la méthode hyperbolique et celle par construction graphique d'Asoaka présentent une bonne concordance.

\section{Généralisation de la loi de consolidation}

La détermination de la loi de consolidation constitue une première étape dans l'élaboration d'une nouvelle méthode de calcul des tassements des sols compressibles. La seconde étape est celle de la généralisation de cette loi à n'importe quelle longueur de chemin hydraulique.

Dans un sol donné soumis aux même conditions de chargement, les vitesses de déformations initiales varient en sens inverse des chemins hydrauliques puissance $m$ soit :

On introduit le temps adimensionnel $t^{*}$ défini comme le rapport des vitesses de déformations initiales pour des échantillons de sol soumis aux même conditions de chargement avec deux longueurs de chemin hydraulique respectives $h_{1}$ et $h_{2}$. Ce facteur est donc inversement proportionnel au rapport des chemins hydrauliques puissance $m$, soit (6) :

$$
t^{*}=\frac{\varepsilon_{\dot{0}_{1}}}{\varepsilon_{\dot{0}_{2}}}=\left(\frac{h_{2}}{h_{1}}\right)^{m}
$$

La détermination de l'exposant $m$ se fait par la conduite des essais œdométriques avec deux longueurs de chemin hydraulique différentes. Cela peut se faire en utilisant le même œdomètre en drainage unilatéral ou bilatéral ou en disposant de deux œdomètres présentant des hauteurs d'échantillon différentes 2 et $4 \mathrm{~cm}$ par exemple.

Dans ces conditions, la généralisation de la loi de consolidation à n'importe quelle longueur de chemin hydraulique $h$ s'écrit (7):

$$
\varepsilon_{t}=\frac{t}{\frac{t}{\varepsilon_{\dot{0}_{1}}}+\frac{t}{\varepsilon_{\infty}}} \quad t=\left(\frac{h}{h_{1}}\right)^{m} .
$$

Les essais ont été effectués avec la vase et le ghassoul en drainage unilatéral et bilatéral avec un chargement de $100 \mathrm{kPa}$ (Fig. 16a). 

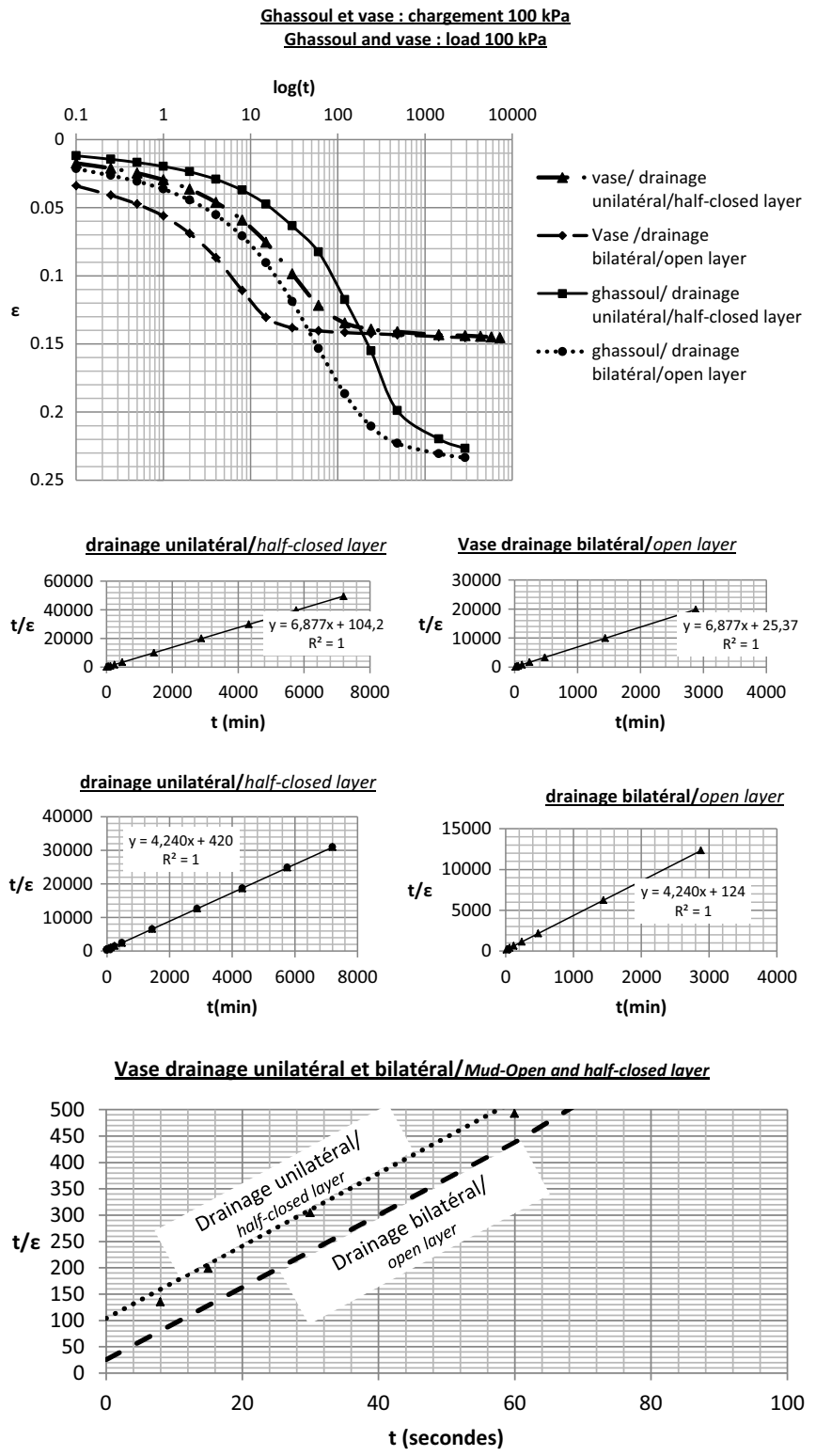

Fig. 17. a: Chargement à $100 \mathrm{kPa}$ de la vase et du ghassoul en drainage unilatéral et bilatéral. Durée 5 jours ; b-c : Consolidation de la vase en drainage unilatéral et bilatéral; d-e: Consolidation du ghassoul en drainage unilatéral et bilatéral ; $\mathrm{f}$ : Vitesse de déformation initiale et longueur du chemin hydraulique pour la vase.

Fig. 17. a: Loading at $100 \mathrm{kPa}$ of mud and ghassoul in open and halfclosed layer. Duration 5 days; $b-c$ : Consolidation of the mud in open and half-closed layer; $d-e$ : Consolidation of the ghassoul in open and half-closed layer; $f$ : Initial strain rate and hydraulic length for mud.

La valeur du coefficient $m$ calculée dans le cas de la vase est de 2,04; elle est en conformité avec la théorie usuelle (Fig. 16b, c et Tab. 6a). En revanche, pour le ghassoul, la valeur du coefficient $m$ est de 1,76 , ce qui confirme que l'application de la théorie usuelle dans ce cas ne serait pas pertinente (Fig. 16d, e et Tab. 6b).

On obtient ainsi les paramètres de la transposition $t^{*}$ et $m$ pour la vase (Tab. 6a).
Il faut noter que, pour un sol donné, la valeur de la déformation finale $\left(\varepsilon_{\infty}\right)$ est identique pour les mêmes conditions de chargement et que seules varient les vitesses initiales de déformation. Celles-ci décroissent en fonction de la longueur du chemin hydraulique. Ainsi, en représentation $t /$ $\varepsilon=f(t)$, les droites de consolidation sont parallèles et seule l'ordonnée à l'origine est variable (Fig. 16f, cas de la vase en drainage unilatéral et bilatéral).

\section{Applications de la loi hyperbolique}

\subsection{Calcul du $c_{v}$ par la méthode hyperbolique}

Ce calcul est effectué en utilisant la fonction hyperbolique pour calculer le $t_{50}$ correspondant à $\frac{\varepsilon_{\infty}}{2}$; le $t_{50}$ est égal au rapport de la déformation finale $\varepsilon_{\infty}$ sur la vitesse de déformation initiale $\dot{\varepsilon}_{0}$. soit : $t_{50}=\frac{\varepsilon^{\infty}}{\dot{\varepsilon}_{0}}$. Le calcul du $c_{v}$ pourra être effectué en utilisant la relation (4). Le tableau 7 présente les résultats des $c_{v}$ calculés par cette méthode et par celle de Casagrande dans le cas de la vase en drainage unilatéral et bilatéral pour un palier de chargement de 0 à $100 \mathrm{kPa}$.

Les valeurs des coefficients de consolidation $c_{v}$ calculées par les deux méthodes sont parfaitement comparables.

\subsection{Estimation des tassements finaux sous remblais de la deuxième rocade urbaine Rabat-Salé}

Dans le cadre du projet de la réalisation de la deuxième rocade urbaine de Rabat-Salé/Lot 2, et compte tenu de la nature du sol d'assises constitué de vase compressible, il a été procédé à son préchargement afin d'accélérer le processus de consolidation. Des tassomètres ont été placés pour suivre l'évolution des tassements générés par les préchargements, en vue notamment de pouvoir apprécier le moment opportun pour procéder au déchargement. Les mesures ont été réalisées durant 8 mois entre le 21/04/2016 et le 23/12/2016. Le préchargement a été effectué d'une manière progressive entre le 21/04/2016 et le 08/08/2016. La hauteur atteinte par le remblai au niveau du tassomètre référencé $125 \mathrm{BTS} 2$ est de 6,5 mètres; elle est de 8 mètres au niveau du tassomètre référencé 129BTS2. L'estimation des tassements finaux $\left(\Delta h_{\text {final }}\right)$ a été faite en utilisant deux méthodes : la méthode hyperbolique et la méthode graphique d'Asoaka. La détermination du tassement final fait l'objet des figure $18 \mathrm{a}$ et $\mathrm{b}$ pour le tassomètre $125 \mathrm{BTS} 2$ et de la figure $18 \mathrm{c}$ et $\mathrm{d}$ pour le tassomètre 129BTS2. Le tableau 8 synthétise les résultats obtenus par les deux méthodes.

Comme on peut le constater dans le tableau 8 , les valeurs des tassements fournies par la méthode graphique d'Asoaka et la méthode hyperbolique sont quasiment identiques. La méthode hyperbolique contrairement à la méthode d'Asoaka, présente toutefois l'avantage d'être facile à mettre en œuvre car elle ne requiert ni régularité des mesures ni la nécessité de procéder à des interpolations.

\section{3 Évolution des tassements du site de Cubzac-les- Ponts (remblai B) d'après Magnan et Baghery (1981)}

Nous avons repris le traitement des tassements par la méthode hyperbolique des mesures effectuées sur le site de Cubzac-les- Ponts (remblai B) (Fig. 19); ces mesures 

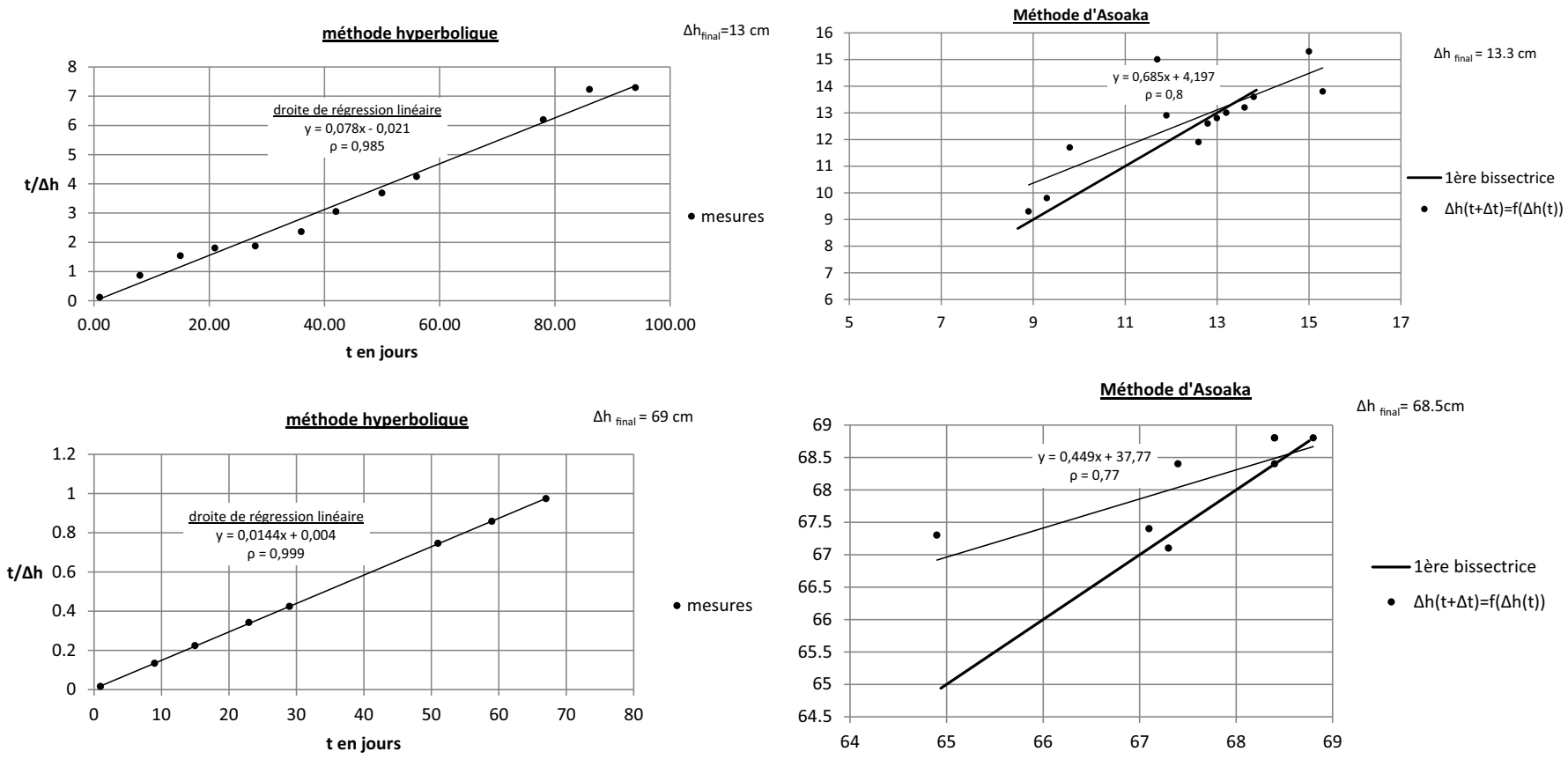

Fig. 18. a : $\triangle h_{\text {final }}$ au 125BTS2 par la méthode hyperbolique ; b : $\triangle h_{\text {final }}$ au 125BTS2 par la méthode d'Asoaka; $: \triangle h_{\text {final }}$ au $129 \mathrm{BTS} 2$ par la méthode hyperbolique; $\mathrm{d}: \triangle h$ final au 129BTS2 par la méthode d'Asoaka.

Fig. 18. a: Final settlement to cell 125BTS2 by the hyperbolic method; b: Final settlement to cell 125BTS2 by the Asoaka method; c: Final settlement to cell129BTS2 by the hyperbolic method; d: Final settlement to cell 129BTS2 by the Asoaka method.

Tableau 4. Ghassoul: déformations mesurées et estimées par les méthodes hyperbolique et d'Asoaka.

Table 4. Ghassoul: deformations measured and estimated by the hyperbolic and Asoaka methods.

\begin{tabular}{llll}
\hline Chargement & $0 \rightarrow 200 \mathrm{kPa}$ & $200 \rightarrow 400 \mathrm{kPa}$ & $400 \rightarrow 600 \mathrm{kPa}$ \\
\hline$\varepsilon_{\infty \text { hyperbolique }}$ & 0,33 & 0,09 & 0,06 \\
$\varepsilon_{\infty \text { Asoaka }}$ & 0,31 & 0,08 & 0,05 \\
$\varepsilon_{24 \mathrm{~h}}$ & 0,29 & 0,08 & 0,05 \\
$\varepsilon_{5 \mathrm{j}}$ & 0,34 & 0,10 & 0,07 \\
\hline
\end{tabular}

Tableau 5. Vase: déformations mesurées et estimées par les méthodes hyperbolique et d'Asoaka.

Table 5. Mud: deformations measured and estimated by the hyperbolic and Asoaka methods.

\begin{tabular}{llll}
\hline Chargement & $0 \rightarrow 200 \mathrm{kPa}$ & $200 \rightarrow 400 \mathrm{kPa}$ & $400 \rightarrow 600 \mathrm{kPa}$ \\
\hline$\varepsilon_{\infty \text { hyperbolique }}$ & 0,27 & 0,05 & 0,03 \\
$\varepsilon_{\infty \text { Asoaka }}$ & 0,27 & 0,05 & 0,03 \\
$\varepsilon_{24 \mathrm{~h}}$ & 0,27 & 0,05 & 0,03 \\
$\varepsilon_{2 \mathrm{j}}$ & 0,27 & 0,05 & 0,03 \\
\hline
\end{tabular}

accompagnaient la présentation d'une méthode approchée pour la prévision des vitesses de tassements des sols fins doués de fluage développée par Magnan et Baghery, qui a fait l'objet d'une publication au Bulletin de Liaison des Laboratoires des Ponts et Chaussées 111 en 1981.

La figure 19 montre qu'il y a un écart significatif entre les mesures des tassements et les calculs effectués par la méthode
Tableau 6a. Paramètres de transposition pour la vase.

Table 6a. Transposition parameters for the mud.

\begin{tabular}{lllllll}
\hline$h_{1}(\mathrm{~mm})$ & $h_{2}(\mathrm{~mm})$ & $h_{2} / h_{1}$ & $\varepsilon_{\dot{0}_{1}}\left(\mathrm{~s}^{-1}\right)$ & $\varepsilon_{\dot{0}_{2}}\left(\mathrm{~s}^{-1}\right)$ & $t^{*}=\frac{{ }^{\varepsilon} \dot{0}_{1}}{\varepsilon_{\dot{0}_{2}}}$ & \\
\hline 10 & 20 & 2,00 & 0,0394 & 0,0096 & 4,10 & 2,04 \\
\hline
\end{tabular}

Tableau 6b. Paramètres de transposition pour le ghassoul.

Table 6b. Transposition parameters for the ghassoul.

\begin{tabular}{lllllll}
$h_{1}(\mathrm{~mm})$ & $h_{2}(\mathrm{~mm})$ & $h_{2} / h_{1}$ & $\varepsilon_{\dot{0}_{1}}\left(\mathrm{~s}^{-1}\right)$ & $\varepsilon_{\dot{0}_{2}}\left(\mathrm{~s}^{-1}\right)$ & $t^{*}=\frac{{ }^{\varepsilon} \dot{0}_{1}}{\varepsilon_{\dot{0}_{2}}}$ & \\
\hline 20 & 10 & 0,50 & 0,0024 & 0,0081 & 0,30 & 1,76 \\
\hline
\end{tabular}

Tableau 7. $C_{\mathrm{v}}$ de Casagrande et $C_{\mathrm{v}}$ hyperbolique dans le cas de la vase en drainage unilatéral et bilatéral.

Table 7. $C_{v}$ of Casagrande method and $C_{v}$ of hyperbolic method in the case of the mud in open and half-closed layer.

\begin{tabular}{lll}
\hline Méthode & \multicolumn{2}{c}{$c_{\mathrm{v}}\left(\mathrm{m}^{2} / \mathrm{s}\right)$} \\
\hline & Drainage unilatéral & Drainage bilatéral \\
Casagrande & $7,49.10^{-8}$ & $1,11.10^{-7}$ \\
Hyperbolique & $8,74.10^{-8}$ & $1,14.10^{-7}$ \\
\hline
\end{tabular}


Tableau 8. Comparaison des tassements finaux.

Table 8. Comparison of final settlements.

\begin{tabular}{llcl}
\hline Tassomètre & \multicolumn{3}{c}{$\Delta h$ final $(\mathrm{cm})$} \\
\hline & Hyperbolique & Asoaka & Mesuré \\
125 BTS2 & 13 & 13,3 & 12,8 \\
129 BTS2 & 69,4 & 68,5 & 69,4 \\
\hline
\end{tabular}

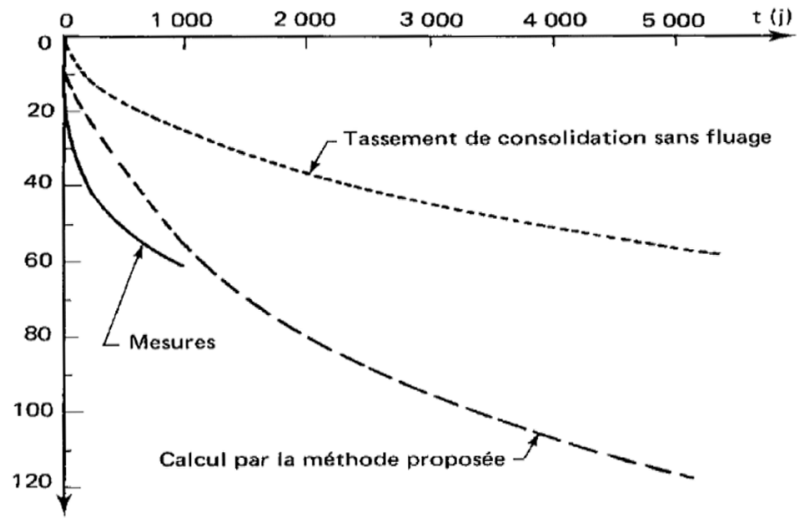

Fig. 19. Analyse des tassements de remblai B du site de Cubzac-lesPonts (d'après Magnan et Baghery).

Fig. 19. Analysis of settlement of $B$ embankment at the Cubzac-lesPonts site (Magnan and Bagher).

proposée. Cela a d'ailleurs été relevé par les auteurs. Toutefois, si l'écart entre les mesures et les calculs semble se réduire avec le temps: il ne s'agit que d'une phase transitoire. En effet l'application de la méthode hyperbolique (Fig. 20) sur les mesures effectuées permet d'extrapoler les mesures à différents horizons de temps. Ainsi, la divergence est notable entre la tendance du modèle de calcul développé et celle des mesures (Fig. 21).

\subsection{Courbe de compressibilité stabilisée et contrainte de préconsolidation}

Note: dans cette section on utilisera indifféremment les plans de représentation ( $\log \sigma, \varepsilon$ [déformation]) et $(\log \sigma$, e [indice des vides]); dans le premier cas l'orientation de l'axe des ordonnées est vers le bas; elle est vers le haut dans le second cas.

Il a été rappelé dans le paragraphe 3 que le temps $t_{100}$ correspond selon la théorie classique à la dissipation totale de la surpression interstitielle et marque la fin de la consolidation primaire; or, les résultats des mesures (Fig. 9a et b) donnent des pressions à $t_{100}$ de $32 \mathrm{kPa}$ pour la vase et de $60 \mathrm{kPa}$ pour le ghassoul. Ceci montre que pour ces expériences le gradient hydraulique entre la base de l'œdomètre et la pierre poreuse supérieure n'est pas nul à $t_{100}$. L'eau continuera donc de s'écouler de l'éprouvette vers la pierre poreuse à la fin de la phase primaire et pendant la phase secondaire telles que définies par la théorie classique de consolidation (Fig. 2). Il peut être conclu que l'équilibre hydraulique n'est donc pas atteint à la fin de la consolidation primaire et que les déformations de la phase secondaire sont dues au départ de

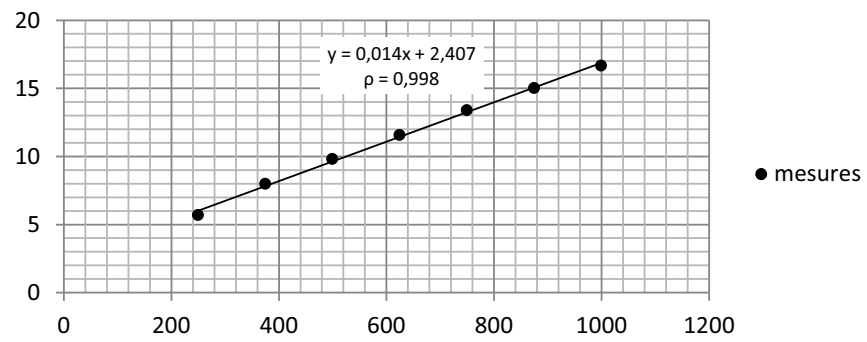

Fig. 20. Application de la méthode hyperbolique aux mesures du site de Cubzac-les- Ponts, remblai B.

Fig. 20. Application of the hyperbolic method to measurements at the Cubzac-les-Ponts site, embankment B.

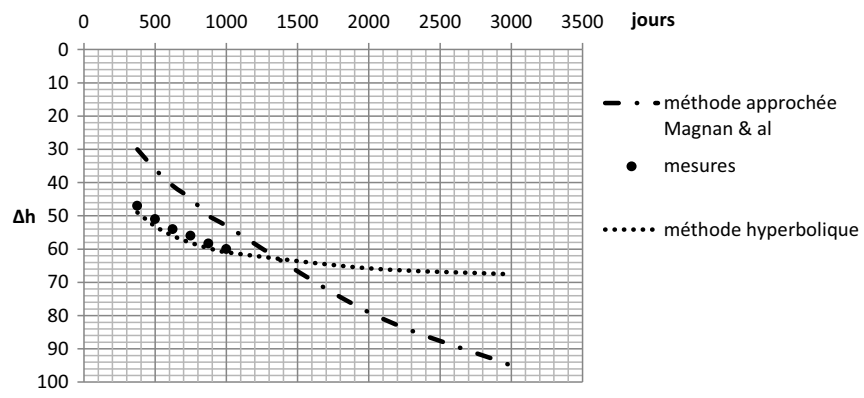

Fig. 21. Tendances au long terme des mesures des tassements du site de Cubzac-Les-Flots, remblai $\mathrm{B}$ et de la méthode approchée de prévisions.

Fig. 21. Long-term trends in Cubzac-Les-Flots settlement measurements, embankment $B$ and the predicted forecast method.

l'eau sous un faible gradient hydraulique. La dépendance de $C_{\alpha}$ (coefficient de fluage) des conditions de drainage (Tab. 4) confirme en outre cette hypothèse. Ainsi, l'état d'équilibre sera atteint quand la surpression interstitielle sera totalement dissipée. La déformation totale de l'éprouvette due à un chargement correspond donc à celle atteinte pour cet état d'équilibre. L'utilisation d'une approche globale pour la prédiction des déformations en fonction du temps est pertinente étant donné que les déformations des phases primaire et secondaire sont régies par le départ de l'eau. Le point de coordonnées $\left(t_{100}, \Delta h_{100}\right)$ marque le basculement entre un régime d'écoulement rapide et un régime d'écoulement lent. Dans le présent article, l'approche empirique basée sur l'extrapolation des points expérimentaux $(\varepsilon, t)$ déduits de l'essai œdométrique a été privilégiée. Les modèles hyperboliques et d'Asoaka ont été utilisés pour ce faire et ont permis d'avoir d'excellents coefficients de détermination $\mathrm{R}^{2}$ avec les points expérimentaux (Fig. 11, 13, 17a et b). Ces deux modèles permettent de prédire la valeur asymptotique de la déformation finale sous une charge donnée $\varepsilon_{\infty}=f(\sigma)$; cette déformation correspondra donc à l'état d'équilibre hydraulique de l'éprouvette.

Les courbes de compressibilité $e=f(\sigma)$, désignées par $t_{\mathrm{d}}$ (où $d$ est la durée de chargement), se présentent sous la forme d'un faisceau de lignes parallèles (Taylor, 1948; Crawford, 1964 ; Bjerrum, 1967) qui se décalent vers le bas au fur et à mesure que la durée de chargement augmente (Fig. 22). 


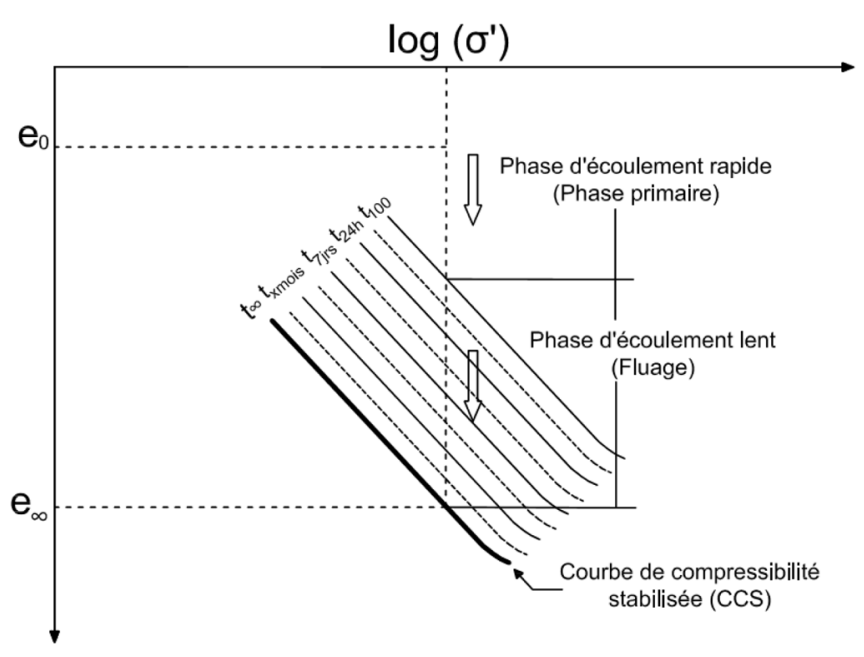

Fig. 22. Courbes de compressibilités et phases d'écoulement.

Fig. 22. Void ratio/log (effective stress) curves and flow phases.

Aussi, sous une charge appliquée $\sigma$, la consolidation du sol suivra une verticale qui interceptera les courbes $t_{\mathrm{d}}$ (Fig. 22). La consolidation s'effectuant avant l'interception de la courbe de consolidation à $t_{100} \mathrm{~s}$ 'opère en phase primaire et donc avec un régime d'écoulement rapide alors que celle qui a lieu au-delà de $t_{100}$ correspond au fluage et un régime d'écoulement lent. Le fluage se poursuivra dans le temps jusqu'à l'interception de la courbe de compressibilité stabilisée (CCS) qui marque la fin de sa dynamique. La CCS correspond à la représentation de couples de valeurs: contrainte appliquée $\left(\log (\sigma) ; e_{\infty}\right)$ et représente l'état d'équilibre hydraulique du sol étudié. Les courbes de consolidation à différentes durées de chargement constituent des états dynamiques dans le processus de consolidation, qui ne prend fin que lorsque la CCS est atteinte.

La courbe de compressibilité stabilisée (CCS) constitue une enveloppe inférieure des courbes de compressibilité à différentes durée de chargement.

La figure 23 présente les points expérimentaux de la déformation en fonction du chargement appliqué pendant une durée de $24 \mathrm{~h}: \varepsilon_{24 \mathrm{~h}}=f(\sigma)$ avec des chargements du ghassoul $0-200 \mathrm{kPa}, 200-400 \mathrm{kPa}$ et $400-600 \mathrm{kPa}$. Sur cette même figure sont représentées les déformations à l'équilibre $\varepsilon_{\infty}$ estimées avec la méthode hyperbolique. Cette courbe représente donc la construction expérimentale de la courbe de compressibilité stabilisée (CCS) définie précédemment. Il est à noter que la déformation à $24 \mathrm{~h}$ représente dans ce cas en moyenne $89 \%$ de la déformation finale, ce qui montre l'intérêt pratique de la construction de la courbe de compressibilité stabilisée (CCS) pour ce sol. Pour la vase, la CCS est quasiconfondue avec celle de $\varepsilon_{24 \mathrm{~h}}$.

Afin d'étudier l'effet d'un déchargement sur le comportement du sol, un échantillon de ghassoul a été ramené à la teneur en eau limite de liquidité $w_{L}$ puis a été soumis à un chargement de $150 \mathrm{kPa}$ durant $24 \mathrm{~h}$ dans un œdomètre en drainage unilatéral. Une série de déchargements successifs a été ensuite appliquée. La durée entre deux déchargements successifs est de $24 \mathrm{~h}$. La figure 24 présente les résultats expérimentaux de déformation en fonction de la charge appliquée. Les courbes $\varepsilon_{24 h}$ et celle de compressibilité stabilisée (CCS) construites précédemment pour ce sol ont été reportées sur cette même

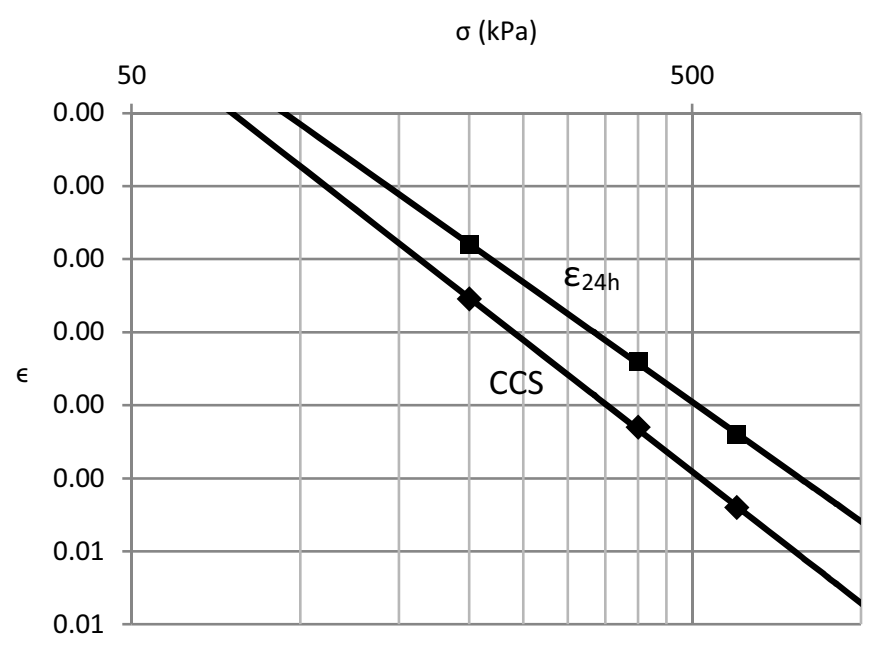

Fig. 23. Déformation à $24 \mathrm{~h}$ et courbe de compressibilité stabilisée (CCS) pour le ghassoul.

Fig. 23. Deformation at $24 \mathrm{~h}$ and Stabilized Compressibility Curve (SCC) for ghassoul.

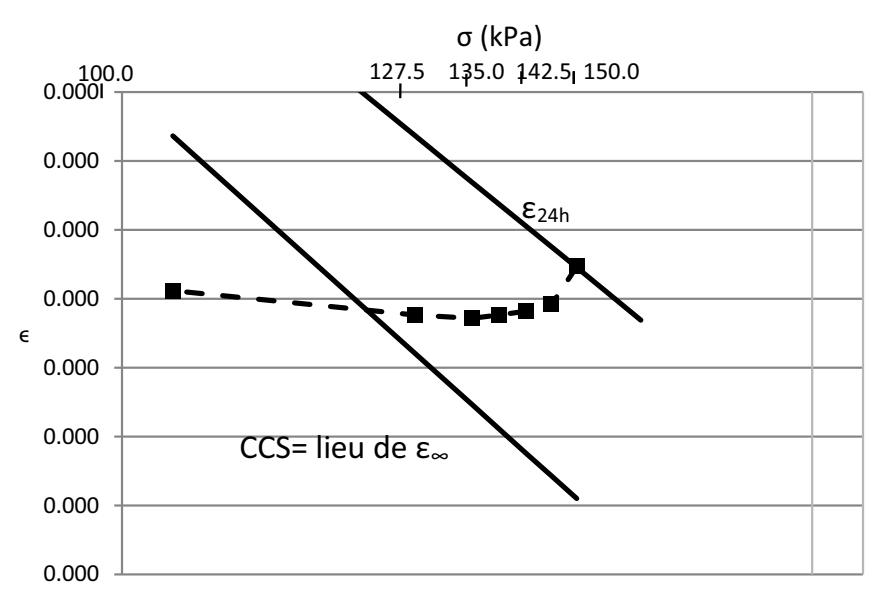

Fig. 24. Déchargement du ghassoul (échelle semi-logarithmique).

Fig. 24. Unloading ghassoul-deformation $(\varepsilon) / \log (\sigma)$.

figure. Il peut être observé que, dans un premier temps et à partir de la courbe $\varepsilon_{24 \mathrm{~h}}$, le sol continue de subir un tassement en dépit des déchargements appliqués. Le gonflement du sol se déclenche pour la contrainte $\sigma=127,5 \mathrm{kPa}$, soit un déchargement de $22,5 \mathrm{kPa}$ par rapport à la contrainte initialement appliquée $(150 \mathrm{kPa})$. On notera que l'amorce du gonflement se fait au voisinage de la courbe de compressibilité stabilisée (CCS). Ainsi, le sol continuera de se tasser à partir de la courbe dynamique $\varepsilon_{24 h}$ et ce tant que la courbe de compressibilité stabilisée (CCS), qui représente l'état d'équilibre hydraulique du sol, n'a pas été atteinte.

$A$ contrario, en effectuant une série de chargements à partir de la courbe de compressibilité stabilisée (CCS) jusqu'à la $t_{24 \mathrm{~h}}$, on obtient d'abord un palier, où les tassements ne sont pas perceptibles, qui intercepte les courbes de compressibilité aux durées de chargement très élevées, puis au fur et à mesure qu'on approche la courbe $t_{24 \mathrm{~h}}$, les tassements augmentent jusqu'à l'intersection avec la courbe. Si le dernier chargement est maintenu, alors la consolidation se fera en régime lent suivant la verticale jusqu'à la CCS. La figure 25 résume le comportement 


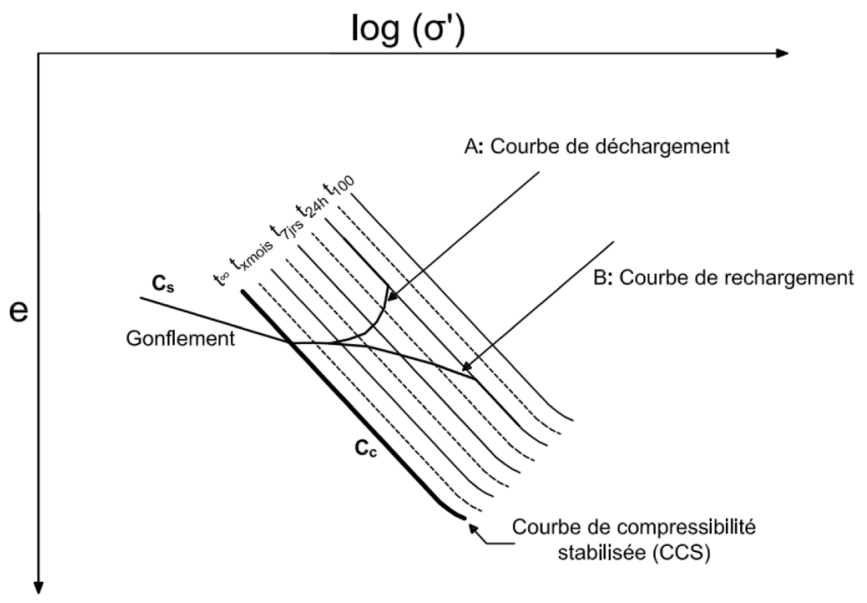

Fig. 25. Courbes de compressibilité et cycle de déchargementsrechargements.

Fig. 25. Void ratio/log (effective stress) curves and unloadingreloading cycles.

d'un sol vis-à-vis d'un déchargement à partir de $t_{24 \mathrm{~h}}$ (courbe $\mathrm{A}$ ) et d'un chargement à partir de la courbe CCS (courbe B).

La contrainte de préconsolidation est usuellement définie comme étant la contrainte verticale maximale sous laquelle le sol s'est consolidé au cours de son histoire. Cette contrainte correspond donc nécessairement à un état d'équilibre final du sol sous une charge appliquée et pour lequel aucune déformation n'a lieu. Ainsi, la courbe de consolidation stabilisée (CCS) telle que définie précédemment constitue le lieu privilégié des contraintes de préconsolidation.

Tout déchargement effectué à partir d'une contrainte de préconsolidation $\sigma$ 'p de cette courbe donnera lieu à un gonflement suivant la pente $C_{\mathrm{s}}$; le retour par rechargement vers $\sigma$ 'p, provoquera un tassement selon une hystérésis de cette même pente. Tout chargement effectué à partir de la CCS provoquera un tassement selon une pente différente de $C_{\mathrm{s}}$ et cette pente croît au fur et à mesure où l'on s'approche de la courbe de $t_{24 \mathrm{~h}}$ (Fig. 25)

Contrairement aux points situés sur la CCS, à partir de tout autre point appartenant à une des courbes dynamiques et non stabilisée $t_{\mathrm{d}}$ (comme la $t_{24 \mathrm{~h}}$, par exemple), les déchargements dont l'amplitude ne dépasse pas la CCS, ne provoquent pas de gonflement mais bien au contraire génèrent des tassements.

Ainsi, les contraintes de préconsolidation portées par la CCS excluent qu'un gain de la contrainte de préconsolidation peut être obtenu par simple fluage sous une contrainte donnée et ce quelle que soit la durée de chargement.

Du point de vue pratique, l'accélération du processus de consolidation peut être réalisée de deux manières :

- la première consiste à opérer un préchargement (contrainte du projet augmentée d'un accroissement $\Delta \sigma$ ) de telle sorte qu'une partie de la déformation soit réalisée en régime rapide, puis à opérer des déchargements jusqu'à atteindre la déformation cible ;

- pour la deuxième méthode, on opère avec le chargement projeté mais on procède à la réduction de la longueur du chemin hydraulique et à l'accroissement du gradient hydraulique par la mise en place de drains verticaux. Une troisième variante consiste à combiner ces deux méthodes.
Le choix d'une de ces méthodes dépendra des contraintes du projet en termes de budget, de délai de réalisation, et de tassements admissibles.

\section{Conclusion}

La dynamique du processus de consolidation est hydraulique et la loi hyperbolique équilatère permet de l'appréhender dans sa globalité. Cette loi est caractérisée par deux paramètres qui sont la déformation finale $\varepsilon_{\infty}$ et la vitesse initiale de déformation $\varepsilon^{\prime}{ }_{0}$. Ces deux paramètres peuvent être déterminés par un simple ajustement graphique d'une droite sur le nuage de points de coordonnées $(t ; t / \varepsilon)$ où $\varepsilon$ est la déformation. La déformation finale est l'inverse de la pente de la droite ajustée ; la vitesse initiale de déformation est l'inverse de l'ordonnée à l'origine. Cette loi peut être transposée à n'importe quelle longueur de chemin hydraulique moyennant des essais à l'œdomètre avec deux longueurs de chemin hydraulique différentes. L'introduction d'un temps adimensionnel $t^{*}$ défini comme étant le rapport des deux vitesses de déformation permet le calcul du facteur de puissance $m$ qui est utilisé pour la généralisation de la loi.

La courbe de compressibilité stabilisée (CCS) constitue une enveloppe inférieure des courbes de compressibilité à différentes durée de chargement; elle constitue le lieu géométrique des contraintes de préconsolidation. Tout déchargement effectué avec des contraintes inférieures à la contrainte de préconsolidation donnera lieu à un gonflement suivant la droite de pente $C_{\mathrm{s}}$, le rechargement vers cette même contrainte de préconsolidation s'accompagnera de tassements et se fera selon une hystérésis de la même pente $C_{\mathrm{s}}$; alors que tout chargement progressif effectué au-delà de la contrainte de préconsolidation provoquera un tassement selon une pente différente de $C_{\mathrm{s}}$ et cette pente croît au fur et à mesure où l'on s'approche de la courbe de $t_{24 \mathrm{~h}}$. Dans ces conditions, il est clair qu'aucun gain de la contrainte de préconsolidation ne peut être obtenu par simple fluage sous une contrainte donnée et ce, quelle que soit la durée de chargement.

\section{Références}

Abbasi N, Rahimi H, Javadi A, Faker A. 2007. Finite difference approach for consolidation with variable compressibility and permeability. Comput Geotech 34 (1): 41-52.

Al Shamrani. 2004. Applying the hyperbolic method and $\mathrm{C}_{\mathrm{a}} / \mathrm{C}_{\mathrm{c}}$ concept for settlement prediction of complex organic-rich soil formations. Eng Geol 77: 17-34.

Amiri SN, Esmaeily A, Mahouti A. 2011. A Realistic Theory of Soils Consolidation. Geo-Frontiers 2011 Conference, Dallas, Texas, pp. 3828-3837.

Asaoka A. 1978. Observational procedure of settlement prediction. Soils Found 18 (4): 87-100.

Baguelin F. 1999. La détermination des tassements finaux de consolidation: une alternative à la méthode d'Asaoka. Rev Fr Geotech 86: 9-17.

Barden L. 1969. Time dependent deformation of normally consolidated clays and peats. ASCE-J Soil Mech Found Div 95 (SM1): 1-31.

Biot MA. 1941. General theory of three dimensional consolidation. Appl Phys 12: 2. 
Bjerrum L. 1967. Seventh Rankine Lecture. Engineering geology of normally- consolidated marine clays as related to settlements of buildings. Geotech 17 (2): 82-118.

Buisman AS Keverling. 1936. Results of long duration settlement tests. Proc., Intern. Conf. on Soil Mech. and Found. Engr., vol. 1, pp. 103-106.

Conte E, Troncone A. 2007. Nonlinear consolidation of thin layers subjected to time-dependent loading. Can Geotech $J 44$ (1): $717-725$.

Crawford Carl B. 1964. Interpretation of the Consolidation Test, Proc., ASCE, vol. 90, No. SM5, pp. 87-102.

Delage P, Lefebvre G. 1984. Study of the structure of a sensitive Champlain clay and of its evolution during consolidation. Can Geotech J 21 (1): 21-35.

Félix B. 1980. Fluage et consolidation unidimensionnelle des sols argileux. Laboratoire central des ponts et chaussées. Rapp recherche LPC 94: 176.

Félix B. 1981. Présentation d'un nouveau modèle rhéologique et de ses applications à la théorie de la consolidation. Bull Liaison Lab Ponts Chaussées 111: 92-103.

Gibson RE, Lo KY. 1961. A theory of consolidation for soils exhibiting secondary compression. Nor Geotech Ins Oslo 41: $1-16$.

Gibson RE, Schiffman RL, Cargill KW. 1981. The theory of onedimensional consolidation of saturated clays, II. Finite nonlinear consolidation of thick homogeneous layers. Can Geotech $J$ 18: 280-293.

Griffiths FJ, Joshi RC. 1991. Change in pore size distribution owing to secondary consolidation of clays. Can Geotech J 28 (1): 20-24.

Koppejan AW. 1948. A formula combining the Terzaghi load compression relationship and the Buisman secular time effect, Proc. 2th. Int. Conference Soil Mechanics and Foundation Engineering, Rotterdam.

Leonards GA, Ramiah BK. 1959. Time effects in the consolidation of clays. In "Time rate of loading in testing soils". ASCE Special Tech Publ 254: 116-130.

Lowe J. 1974. News concepts in consolidation and settlement analysis. ASCE-J Geotech Eng Div 100 (6): 571-612.

Magnan JP. 1986. Modélisation numérique du comportement des argiles molles naturelles, L.C.P.C., Paris. Rapport de Recherche LPC, no 141, 255 p.

Magnan JP, Baghery S. 1981. Une méthode approchée pour la prévision des vitesses de tassement des sols fins doués de fluage. Bull Liaison Lab Ponts Chaussées 111: 87-91.

Magnan JP, Deroy JM. 1980. Analyse graphique des tassements observés sous les ouvrages. Bull Liaison Lab Ponts Chaussées 109: $45-52$.

Magnan JP, Baghery S, Brucy M, Tavenas F. 1979. Étude numérique de la consolidation unidimensionnelle en tenant compte des variations de la perméabilité et de la compressibilité du sol, du fluage et de la non- saturation. Bull Liaison Lab Ponts Chaussées 103: 83-94.

Mesri G. 1973. Coefficient of secondary compression. J Soil Mech Found Div ASCE 99 (SM1): 123-137.

Mesri G, Godlewski PM. 1977. Time- and stress- compressibility interrelationship. J Geotech Eng Div ASCE 103 (GT5): 417-430.

Nakaoka K, Yamamoto S, Hasegawa H, et al. 2004. Long-term consolidation mechanisms based on micro-macro behavior and in situ XRD measurement of basal spacing of clay minerals. Appl Clay Sci 26 (1): 521-533.

Olson RE. 1989. Secondary consolidation. Chaoyang University of Technology, Department of Construction Engineering: Advanced Soil Mechanics.

Philipponnat G, Hubert B. 2011. Fondations et ouvrages en terre. Paris : Eyrolles.

Poskitt TJ. 1969. The consolidation of saturated clay with variable permeability and compressibility. Geotechnique 19(2).

Schlosser F. 1973. Hypothèses et théories pour la prévision des tassements des remblais sur sols compressibles. Bull Liaison Lab Ponts Chaussées $\mathrm{n}^{\mathrm{o}}$ spécial $\mathrm{T}$.

Skempton AW, Bjerrum L. 1957. A contribution to the settlement analysis of foundations on clay. Geotech 7 (4): 168.

Sridharan A, Sreepada Rao A. 1981. Rectangular hyperbola fitting method for one-dimensional consolidation. Geotech Test $J$ 4: $161-168$

Sridharan A, Murthy NS, Prakash K. 1987. Rectangular hyperbola method of consolidation analysis. Geotech 37: 355-368.

Tan S-A, Chew S-H. 1996. Comparaison of the hyperbolic and Asoaka observational method of monitoring consolidation with vertical drains. Soils Found Jpn Geotech Soc. 36(3): 31-42.

Tan TS, Inoue T, Lee SL. 1991. Hyperbolic method for consolidation analysis. J Geotech Eng ASCE 117(11): 1723-1737.

Tavenas F, Brucy M, Magnan JP, La Rochelle P, Roy M. 1979. Analyse critique de la théorie de consolidation unidimensionnelle de Terzaghi. Rev Fr Geotech 5 (7): 29-43.

Taylor DW. 1948. Fundamentals of soil mechanics. New-York, NY: John Wiley \& Sons.

Taylor DW, Merchant W. 1940. Theory of lays consolidation accounting for secondary compression. J Math Phys 19 (3): 167-185.

Terzaghi K. 1923. Die Berechnung der Durchlässigkeit des Tones aus dem Verlauf der hydrodynamischen Spannungserscheinungen. Akademie der Wissenschaften, Wien, Sitzungsberichte, Math. Naturwiss. KJasse, part. Ii-a, vol. 132 (3/4), pp. 125-138.

Thompson James B, Palmer LA. 1951. Report of Consolidation Tests with Peat, ASTM STP No 126, pp. 4-8.

Vasseur G, Djeran-Maigre I, Grunberg D, Rousset G, Tessier D. 1995. Evolution of structural and physical parameters of clays during experimental compaction. Mar Pet Geol 12 (8): 941-954.

Wang YH, Xu D. 2007. Dual porosity and secondary consolidation. $J$ Geotech Geoenvironmental Eng 133 (7): 793-801.

Citation de l'article : Mohamed Ayeb, Mohamed Benechebli, Youssef Ayeb. Nouvelle approche d'analyse de la consolidation unidimensionnelle des sols argileux. Rev. Fr. Geotech. 2017, 153,2. 